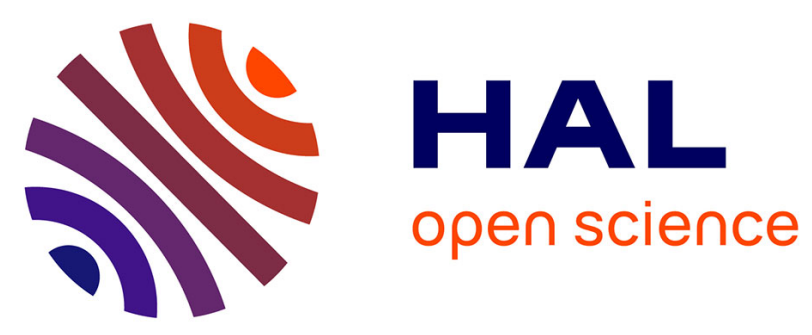

\title{
EXPERIMENTAL EVIDENCE AND PHYSICAL IMPLICATIONS OF THE TIME EVOLUTION ALONG THE MASS ASYMMETRY MODE IN HEAVY ION REACTIONS
}

L. Moretto, R. Schmitt

\section{To cite this version:}

L. Moretto, R. Schmitt. EXPERIMENTAL EVIDENCE AND PHYSICAL IMPLICATIONS OF THE TIME EVOLUTION ALONG THE MASS ASYMMETRY MODE IN HEAVY ION REACTIONS. Journal de Physique Colloques, 1976, 37 (C5), pp.C5-109-C5-140. 10.1051/jphyscol:1976507 . jpa00216587

\section{HAL Id: jpa-00216587 https://hal.science/jpa-00216587}

Submitted on 1 Jan 1976

HAL is a multi-disciplinary open access archive for the deposit and dissemination of scientific research documents, whether they are published or not. The documents may come from teaching and research institutions in France or abroad, or from public or private research centers.
L'archive ouverte pluridisciplinaire HAL, est destinée au dépôt et à la diffusion de documents scientifiques de niveau recherche, publiés ou non, émanant des établissements d'enseignement et de recherche français ou étrangers, des laboratoires publics ou privés. 
EXPERIMENTAL EVIDENCE AND PHYSICAL IMPLICATIONS OF THE TIME EVOLUTION ALONG THE MASS ASYMMETRY MODE IN HEAVY ION REACTIONS*

\section{G. Moretto ${ }^{+}$and R. Schmitt}

Nuclear Science Division, Lawrence Berkeley Laboratory, and Department of Chemistry, University of California, Berkeley, California 94720

Résumé : Des caractéristiques expérimentales complexes sont associées aux distributions de masse ou charge et aux distributions angulaires en fonction de la masse ou de la charge du fragment. Ellies prouvent l'existence d'une structure intermédiaire, ou complexe intermédiaire, dont l'asymétrie de masse êvolue en fonction du temps. De fortes preuves indirectes suggèrent que cette évolution avec le temps correspond à une diffusion et peut être décrite en termes d'Equation Maltresse ou d'Equation de Fokker-Planck. Il est établi expérimentalement que la distribution de masse est large pour les grands rapports $E / B$ - où $E$ est l'énergie dans le centre de masse et $B$ la barrière d'interaction - et étroite avec un maximum à la masse du projectile (et de la cible) pour les petits rapports $E / B$. Ceci est interprété conme étant dû à une augmentation du temps de vie du complexe avec E. Pour les temps de vie courts, le système a peu de temps pour modirier son asymétrie de masse et donne donc naissance à des distributions de masse assez étroites et centrées au voisinage de la masse du projectile (et de la cible). Pour les temps de vie longs, le degré de liberté asymétrie de masse est en voie d'équilibre, ce qui conduit à de très larges distributions de masse. De la même façon, le maximum des distributions angulaires semble se déplacer depuis un angle éloigné de $0^{\circ}$ (pic sur le côté) jusqu'à un angle très proche de $0^{\circ}$ (pic en avant) lorsque $E / B$ crôt. Ceci est interprété comme étant dû à une transition depuis un régime où le temps de vie court et la vitesse angulaire faible ne permettent pas au système de tourner plus loin que $0^{\circ}$, jusqu'à un régime à temps de vie long et vitesse angulaire élevée, qui conduit à une rotation importante du systême sur luimême et à une émission au-delà de $0^{\circ}$. Lorsque la charge nucléaire du produit s'éloigne de celle du projectile, l'évolution depuis le pic sur le côté jusqu'au pic en avant est due au décalage de tenps introduit par la diffusion dans la population des fragments les plus éloignés en Z du projectile. La variation des distributions angulaires et de charge avec l'énergie cinétique du fragment permet de relier l'amortissement en énergie à l'amortissemert en asymétrie de masse. Des calculs théoriques basés sur les modèles de diffusion permettent de bien rendre compte des distributions angulaires et de masse, et aussi d'obtenir des probabilités de transition et des coefficients de Fokker-Planck. La validité des différentes méthodes d'analyse est discutée.

Abstract: The complex experimental features associated with the mass or charge distributions, and with the angular distributions as a function of fragment mass or charge, are interpreted as evidence of an intermediate structure, or intermediate complex, evolving in time along the mass asymmetry mode. Strong circumstantial evidence suggests that this time evolution is diffusive in nature and can be described in terms of the Master Equation or the Fokker-Planck Equation. The experimental evidence of broad mass distributions for large ratios $E / B$, where $E$ is the center of mass energy and $B$ is the interaction barrier, and narrow mass distributions peaked at the projectile and target mass for small ratios $E / B$, is interpreted as due to an increasing lifetime of the complex with energy. For short lifetimes, the system has little time to evolve in mass asymmetry and gives rise to rather narrow distributions centered about the target and projectile mass. For long lifetimes the system undergoes extensive relaxation in mass asymmetry and gives rise to very broad mass distributions. Similarly the angular distributions seem to evolve from side peaked to forward peaked with increasing E/B. This is interpreted as due to a transition from a short lifetime-slow angular velocity regime which does not allow for orbiting beyond $0^{\circ}$, to a long lifetime-large angular velocity regime which produces orbiting past $0^{\circ}$. The evolution from side peaking to forward peaking in the same reaction as one moves away in $Z$ from the projectile is interpreted as 
due to the time lag introduced by diffusion in the population of fragments farther removed in $Z$ from the projectile. The variation of charge and angular distribution with the fragment kinetic energy allows one to connect the energy relaxation to the mass asymmetry relaxation. Theoretical calculations based on diffusion models allow one to fit mass and angular distributions as well as to extract transition probabilities and Fokker-Planck coefficients. The reliability of various methods of analysis is discussed.

\section{INTRODUCTION}

It appears that the accelerator development has occurred along a sound pedagogical line, well suited to our education in nuclear physics. Early machines provided us with simple projectiles which had the merit of mainly inducing two kinds of nuclear reaction: direct reactions on the one hand, and compound nuclear reactions on the other. Both kinds were diligently studied by our parents in science.

The direct reactions, it was learned, portray a strong dynamical coupling between entrance and exit channels, their degree of inelasticity is minimal, and very few degrees of freedom of the target are excited. Then the great chapter of particle spectroscopy was written, and the single particle structure of nuclei (shell structure), with the added refinement of residual interactions, was revealed.

The compound nucleus reactions, on the contrary, told quite a different story. A complete decoupling between entrance and exit channels was observed, together with an extreme degree of inelasticity and the involvement of all of the nuclear degrees of freedom. The compound nucleus was then postulated as a long-lived intermediate in which all the degrees of freedom attain statistical equilibrium. The chapter of the statistical nuclear properties was then written with all the corollaries of statistical distributions, statistical decay, evaporation, etc.

It seems natural that, after having received such primers in nuclear physics, we should enter a new, more comprehensive field which bridges the simplicity of the states explored by direct reactions to the complexity of the compound states. Such a connection involves the great absent in early nuclear physics, the time. Heavy ion reactions do in fact reveal a sequence of patterns whose connection is unmistakably the time. In this sequence, the relatively simple entrance channel configurations appear to evolve into more and more complex configurations, approaching, with variable degree, the ultimate statistical distributions.

Three collective degrees of freedom are particularly noticeable in heavy ion reactions for their various stages of relaxation: the relative distance of the fragments, the neutron-to-proton ratio, and the mass asymmetry. In the first degree of freedom, all the initial kinetic energy appears to relax into the internal degree of freedom through the action of viscous forces. Such a dissipative process is so immediately visible, and involves such a large fraction of the total cross section, that special names have been created for it, like deep inelastic, strongly damped or relaxed [1-4], even though differences are frequently seen in the degree of relaxation associated with the other degrees of freedom. The energy dissipation is a relatively fast process, and has relatively little overlap with the slower relaxation processes associated with other degrees of freedom.

The second degree of freedom, the neutron-toproton ratio, also appears to equilibrate very fast, so that its relaxation seems to proceed essentially at constant mass asymmetry [5-7].

The relaxation processes associated with the first two degrees of freedom have been treated at length in Galin's talk. So we shall concentrate essentially on the features associated with the third degree of freedom, the mass asymmetry. This degree of freedom is rather slow in its time evolution, so that in many cases its relaxation occurs, while other degrees of freedom have already attained their equilibrium distributions: in other words, the kinetic energy has been mostly dissipated into the internal degrees of freedom, giving rise to a "warm" system; the neutron-toproton ratio has been balanced; etc.

Such a system, nearly equitibrated in many degrees of freedom, and slowly evolving along the 
mass asymmetry degree of freedom, we 1ike to call "intermediate complex" in analogy with the intermediate complex of chemical reactions [8-11].

Various facts seem to support the existence of the intermediate complex. For instance, the great amount of cross section concentrated at low kinetic energies shows that in general there is enough time for the kinetic energy to relax. Furthermore, the spreading of the $Z$ distribution seems to occur mostly while the kinetic energy is very low, if not completely thermalized. This means that the mass asymmetry mode relaxes slowly.

This general picture suggests that the evolution along the mass asymmetry is "creepy", or dominated by the viscous forces. Apparently a continuous readjustment in the equilibrium conditions is possible as the system moves along the mass asymmetry coordinate. In this case memory effects are unimportant, and the time evolution can well be described in terms of a diffusion mechanism $[8,9,11-13]$.

An important aspect of the intermediate complex is its decay time, which controls the extent of relaxation observed for the various degrees of freedom. In the lighter systems it is not clear whether the decay of the complex is statistically or dynamically controlled. In the heavier systems the evidence seems to indicate that the decay time is more a dynamical quantity than a statistical quantity. Therefore, it appears that the prevailing regime is that of nonequi]ibrium statistical mechanics for some degrees of freedom, and almost purely dynamical for others.

We shall divide what follows into four sections. In the first section we shall give a qualitative justification for the use of a diffusion model applied to the evolution in mass asymmetry. The Master Equation will be explicitly written down for this process, the corresponding Fokker-Planck Equation will be considered, and the drift and spread coefficients will be related to the transition probabilities. The possibility of a direct use of the Fokker-Planck Equation in order to analyze the mean displacement and the widths of the experimental distributions will be discussed. The alternate and more rigorous procedure of coupling the Master Equation or the Fokker-Planck Equation to the dynamics of other degrees of freedom will be presented.

In the second section, the experimental data on the mass (or charge) distribution will be presented. The dependence of these distributions on angle and energy windows will be shown and the physical implications will be discussed.

Similarly in the third section, the angular distributions and their dependence upon $Z$ and energy windows will be presented. The time factor controlling the features of the angular distributions will be discussed.

In the brief fourth section, examples of theoretical calculations will be compared with experiment, and numerical values for the decay times and diffusion coefficients obtained from various sources will be discussed.

SECTION I. THEORETICAL CONSIDERATIONS

Lagrangian and Diffusive Approaches to the Description of Time Dependent Processes

The fission process has been one of the first nuclear processes to be treated in a timedependent fashion. In a couple of brilliant papers, Nix $[14,15]$ described the time evolution from saddle to scission point by introducing a Lagrangian in the collective variables. The liquid drop model was used for the potential energy, and an irrotational flow was assumed for the inertia tensor. In principle the extension of these calculations to heavy ion reactions is trivial. It is not clear, however, if this approach is sufficiently general.

The Lagrangian approach establishes a pointto-point correspondence between the initial and the final phase space and thus is completely deterministic. More clearly, the trajectory, in a Lagrangian formulation, is a well defined entity, and for a given initial condition, or point in phase space, there is one and only one trajectory. The final distributions depend exclusively upon the distributions of initial conditions.

While such an approach, generalized by the introduction of the Rayleigh dissipation function to handle viscous forces, may be applicable under certain circumstances [16-24], it actually has serious deficiencies which may prevent its success in describing the overall evolution of the shape parameters in heavy ion reactions. The shortcomings of the Lagrangian approach to the description of a manybody system arise from the neglect of the internal degrees of freedom [11]. If one considers an ensemble of systems, all having the same initial conditions in collective phase space, their time evolution will be described by a 
diverging set of trajectories, rather than by a single trajectory, because of the unspecified initial conditions for the internal degrees of freedom. Therefore, an accurate description of the time evolution of the ensemble cannot be completely deterministic, but must also contain the statistical influence of the internal degrees of freedom in determining the distribution of the elements of the ensemble in collective phase space. One can look at this problem more concretely as follows. After the kinetic energy is dissipated, the intermediate complex has a temperature that may range, typically, between 1 and $4 \mathrm{MeV}$. While this system follows a Lagrangian trajectory in collective phase space with a few tens of MeV kinetic energy, it is subjected to random Brownian impulses which are comparable to the momentum of the system along the collective coordinate. As a consequence the Lagrangian trajectory is seriously perturbed, so that the actual trajectories of the various elements of the ensemble tend to diverge.

Nörenberg will show in greater detail under which conditions the use of the Master Equation can be justified. We shall assume that the Master Equation is indeed a suitable tool to describe the evolution in time of the mass distribution and we shall apply it directly to our problem.

Application of the Master Equation to the Diffusion Along the Mass Asymmetry Coordinate

Let us label the asymmetry of the intermediate complex by means of the atomic number $z$ of one of the two fragments in contact. Furthermore, let us assume that the complex evolves in time through configurations of different asymmetries by means of a stochastic process, as required by the Master Equation. Then, the time evolution of the population $\phi(Z, t)$ can be written as [8]:

$$
\dot{\phi}(z, t)=\sum_{z^{\prime}}\left(\Lambda_{z z^{\prime}} \phi\left(z^{\prime}, t\right)-\Lambda_{\left.z^{\prime} z \phi(z, t)\right)}\right.
$$

where $\dot{\phi}$ is the time-derivative of the population and $\Lambda_{z, z^{\prime}}, \Lambda_{z^{\prime} z}$ are the macroscopic transition probabilities coupling the configurations $Z^{\prime}$ and $Z$. The form of $\Lambda_{z z^{\prime}}$ and $\Lambda_{z^{\prime} z}$ and the range of $z^{\prime}$ 's over which one must extend the sum, must be described. Without any loss of generality we can rewrite:

$$
\begin{aligned}
& \Lambda_{z z^{\prime}}=\lambda_{z z^{\prime}} \rho_{z} \\
& \Lambda_{z^{\prime} z}=\lambda_{z^{\prime} z^{\prime} z^{\prime}} \\
& \lambda_{z z^{\prime}}=\lambda_{z^{\prime} z},
\end{aligned}
$$

where $\lambda_{z z}$, is the microscopic transition probability (which is symmetric because of microscopic reversibility); and $\rho_{z}, \rho_{z}$, are the statistical weights of the macroscopic configurations. The latter quantities can be identified with the level densities of the complex:

$$
\rho_{z}=\rho\left(E-V_{z}\right),
$$

where $E$ is the total energy of the system; and $V_{z}$ is its potential energy (including rotational energy). For small $v_{z}$ one can expand the level density as follows [8]:

$$
\rho\left(E-V_{z}\right)=\rho(E) \exp -V_{z} / T
$$

where

$$
T^{-1}=\left.\frac{\partial \ln \rho}{\partial x}\right|_{x=E}
$$

The quantity $T$ can be identified with the thermodynamic temperature.

The quantity $\lambda_{z z}$, can be written as [8]:

$$
\lambda_{z z^{\prime}}=\frac{k}{\sqrt{\rho z^{\rho} z^{\prime}}} f,
$$

where $k$ is a velocity of the order of the Fermi velocity, and $f$ is a form factor which we take to be equal to the window open between the two fragments:

$$
f=2 \pi \frac{R_{1} R_{2}}{R_{1}+R_{2}} d \quad(d=1.0 \mathrm{fm}) .
$$

Notice how the mean level density contained in the denominator of $\lambda_{z z}$ allows the macroscopic transition probability $\Lambda_{z z}$, to remain of the order of $k f$. The sum can be restricted to values of $Z=Z \pm 1$ in the spirit of the independent particle model.

The master equation can now be written as:

$$
\begin{aligned}
\dot{\phi}_{z}=\sum_{z^{i}=z \pm 1} & \frac{k f}{\exp -\frac{V_{z}+V_{z^{\prime}}}{2 T}} \\
& \times\left(\phi_{z^{\prime}} \exp -V_{z^{\prime}} / T-\phi_{z} \exp -V_{z^{\prime}} / T\right)
\end{aligned}
$$

In this form, the equation has been used by us to calculate the $Z$ distribution and the angular distribution of the fragments emitted in the reactions $\mathrm{Ag}+\mathrm{Ar}$ and $\mathrm{Au}+\mathrm{Kr}[8,26]$. 


\section{The Fokker-Planck Approximation}

The implication of the Master Equation as written above, can be better appreciated if we consider it in its approximate Fokker-Planck form:

$$
\begin{aligned}
\dot{\phi}(Z, t)=-\frac{\partial}{\partial Z}\left[\mu_{1}(Z)\right. & \phi(Z, t)] \\
& +\frac{1}{2} \frac{\partial^{2}}{\partial z^{2}}\left[\mu_{2}(z) \phi(z, t)\right] .
\end{aligned}
$$

The quantities $\mu_{1}(Z)$ and $\mu_{2}(z)$ are given by:

$$
\begin{aligned}
& \mu_{1}=\int_{z-1}^{z+1}\left(z^{\prime}-z\right) \Lambda_{z^{\prime} z} d z^{\prime} \\
& \mu_{2}=\int_{z-1}^{z+1}\left(z^{\prime}-z\right)^{2} \Lambda_{z^{\prime} z^{\prime}} d z^{\prime}
\end{aligned}
$$

and can be calculated explicitly.

The transition probability $\Lambda_{z z}$, can be written as follows:

$$
\Lambda_{z z^{\prime}}=\kappa f \exp \frac{V_{z}-V_{z^{\prime}}}{2 T} \text {. }
$$

The potential $V_{z}$, can be expanded as:

$$
v_{z^{\prime}}=v_{z}+\frac{\partial v_{z}}{\partial z} h=v_{z}+v_{z}^{\prime} h
$$

and we have:

$$
\Lambda_{z z^{\prime}}=k f \exp \left(-\frac{V_{z}^{\prime} h}{2 T}\right) .
$$

Finally, we can calculate $\mu_{1}$ and $\mu_{2}$ :

$$
\mu_{1}(z)=\kappa f\left(\frac{8 T^{2}}{V_{z}^{2}} \sinh \frac{V_{z}^{\prime}}{2 T}-\frac{4 T}{V_{z}^{\prime}} \cosh \frac{V_{z}^{\prime}}{2 T}\right)
$$

$\mu_{2}(Z)$

$=k f\left(\frac{4 T}{V_{z}^{\prime}} \sinh \frac{V_{z}^{\prime}}{2 T}-\frac{16 T^{2}}{V_{z}^{\prime 2}} \cosh \frac{V_{z}^{\prime}}{2 T}+\frac{32 T^{3}}{V_{z}^{\prime 3}} \sinh \frac{V_{z}^{\prime}}{2 T}\right)$.

In the fairly common limit of small $\frac{V_{z}^{\prime}}{2 T}$, we obtain:

$$
\begin{array}{ll}
\mu_{1}(z)=-\kappa f \frac{1}{3} \frac{V_{z}^{\prime}}{T} & \\
\mu_{2}(z)=\frac{2}{3} \kappa f & \mu_{2} / \mu_{1}=\frac{2 T}{V_{z}^{\prime}} .
\end{array}
$$

From the definition of $\mu_{7}$ one sees that it corresponds to the average displacement in $Z$ per unit time. In other words, it represents the average velocity along $Z$. Also one can notice that such a velocity depends only upon the coordinate $Z$ and does not depend on any initial velocity. Therefore, it can be identified with the limiting velocity $(t \rightarrow \infty) v=\frac{C}{K}$ associated with the differential equation:

$$
m \dot{v}=-k v+c,
$$

where $m$ is the mass, $k$ is the viscosity coefficient, and $c$ is the force. The viscosity coefficient is then:

$$
K=\frac{3 T}{K f}
$$

Notice that $\kappa$ is nearly independent of temperature for a highly degenerate Fermi gas:

$$
\kappa=\kappa_{0}\left(1+\frac{\pi^{2}}{6} \frac{T^{2}}{\varepsilon_{F}^{2}}+\ldots\right),
$$

where $\varepsilon_{F}$ is the Fermi energy. It follows that the viscosity coefficient is proportional to $T$ for $T \ll \varepsilon_{F}$ and proportional to $T^{T / 2}$ for $T \gg \varepsilon_{F}$. The coefficient $\mu_{2}$ can be rewritten as $\mu_{2}=\frac{2 T}{K}$. In the case $T \ll \varepsilon_{F}, \mu_{2}$ does not depend on temperature.

Let us now consider two simple cases of practical importance. The first case is that of a constant force, which corresponds a to constant slope in the driving potential, and constant temperature. For the initial condition $\phi\left(Z_{0}, 0\right)=\delta\left(Z-Z_{0}\right)$, the solution of the FokkerPlanck equation is:

$$
\phi(z, t)=\frac{1}{\sqrt{2 \pi \mu_{2} t}} \exp -\frac{\left(z_{0}-\mu_{1} t\right)^{2}}{2 \mu_{2} t} .
$$

This is a Gaussian whose centroid moves with velocity $\mu_{1}$ and whose second moment is:

$$
\sigma^{2}=\mu_{2} t
$$

A conceivable experimental test of the applicability of the above equation is a plot of the first moment of the experimental distribution vs. the second moment. Since both are proportional to the time $t$, such plot should be linear. From such an analysis the ratio $\mu_{1} / \mu_{2}$ be obtained. 25

The explicit knowledge of the time $t$ leads to the independent determination of $\mu_{1}$ and $\mu_{2}$. It has been proposed that the time can be inferred from the knowledge of the angular position $\theta$ of the cross section peak in a Wilczynski plot: 12,13

$$
t=\left(\theta-\theta_{0}\right) \frac{\mathscr{g}}{I},
$$


where $\mathcal{J}$ is the moment of inertia; and I the angular momentum. Since both $\theta_{0}$ and $\mathcal{Y}$ depend on $I$, only a detailed knowledge of the deflection function can lead to an accurate determination of the time.

Alternatively, one can rely upon the energy loss in order to obtain the time. For a viscous dissipation in the relative motion of target and projectile one obtains:

$$
v=v_{0} e^{-\frac{K}{m} t}, \text { or } \ln \frac{E_{0}}{E}=\frac{2 K}{m} t,
$$

or, in order to account for Coulomb and rotational energies $E_{C}$ and $E_{R}$,

$$
\ln \frac{E_{0}-E_{C}-E_{R}}{E-E_{C}-E_{R}}=\frac{2 K}{m} t \text {. }
$$

This expression can be of some use in relating the energy dissipation along the relative motion coordinate to the diffusion along. the mass asymmetry coordinate. However, the strong I dependence of this expression and the uncertainty about $K$ and make its use doubtful.

The second case of interest is that of the diffusion driven by a parabolic potential:

$$
V=\frac{c}{2}\left(z-z_{s}\right)^{2}=\frac{1}{2} c h^{2}
$$

In many practical cases the potential energy along the mass asymmetry coordinate is nearly harmonic close to the symmetric value $Z_{s}$. If the initial condition is:

$$
\phi\left(h_{0}, 0\right)=\delta\left(h-h_{0}\right),
$$

the Fokker-Planck Equation gives:

$$
\begin{aligned}
\phi(h, t)= & \sqrt{\frac{c}{2 \pi T\left(1-\exp \left(-\frac{2 c t}{K}\right)\right)}} \\
& \quad \times \exp \frac{c\left(h-h_{0} \exp \left(-\frac{c}{K} t\right)\right)^{2}}{2 T\left(1-\exp \left(-\frac{2 c t}{K}\right)\right)}
\end{aligned}
$$

Notice that the solution is a Gaussian whose centroid moves following the equation:

$$
\ddot{h}+\frac{K}{m} \dot{h}+\frac{c}{m} h=0
$$

which, in the limit $\frac{K}{m} \approx \frac{c}{m} \gg 1$ has the solution:

$$
h=h_{0} e^{-\frac{c}{k} t}
$$

A limitation of this formalism is associated with the constant temperature T. Even when the potential is parabolic, the energy difference between the injection point and symmetry may be so large that the temperature along the parabola changes substantiaî̄y.

Application of the Master Equation to the Evaluation of Cross Sections and Angular Distributions

Moretto and Sventek $[8,11,26]$ have performed diffusion calculations for some of the reactions studied experimentally. A most important quantity for this calculation is the potential energy of the intermediate complex as a function of $Z$ for each partial \& wave. The potential energy has been calculated assuming the shape of two touching spheres for the complex. The energies are computed by means of the liquid drop model:

$$
v_{z}=E_{L D}(Z)+E_{\text {LD }}\left(Z_{\text {tot }}-Z\right)+v_{\text {Cou })}+E_{\text {Rot }} \text {, }
$$

where the first two terms are the liquid drop masses of the two fragments; $V_{\text {Coul }}$ is the Coulomb interaction of the two fragments; and $E_{\text {Rot }}$ is the rotational energy of the complex. Examples of the potential energies and of the populations probabilities as a function of time are shown in Fig. 1. The drift of the distributions from the injection point towards low potential energies is well illustrated. Perhaps more impressive is the spread of the distribution which increases very dramatically with time. This illustrates how a Lagrangian approach might miss a most important feature, namely the spreading of the distribution which, for large times, dominates the picture. It is also important to notice how the angular momentum shifts the Businaro-Gallone mountain with respect to the injection point and how this affects the direction of the drift. This feature is particularly visible in the $\mathrm{Ag}+\mathrm{Ne}$ case.

At this point it is possible to calculate the $Z$ distribution integrated over angle, provided one knows the distribution of lifetimes as a function of impact parameter $b, \Pi(t, b)$ :

$d \sigma / d Z=\int_{0}^{\infty} d t \int_{0}^{\infty} 2 \pi b P(b) \phi_{z}(b, t) \Pi(t, b) d b$

One can go one step farther and evaluate the cross section as a function of $Z$ and $\theta$ directly. The differential cross section can be written as follows $[8,26]$ :

$\frac{\partial^{2} \sigma}{\partial Z \partial \Omega}=\int_{0}^{\infty} d t\left\{\sum_{b} \frac{b P(b)}{\sin \theta \frac{d \theta}{d b}} \phi_{z}(b, t) \Pi(t, b)\right\}$, 
where $P(b)$ is the probability that a collision at impact parameter $b$ leads to a deep inelastic collision. The sum is carried over all the impact parameters $b$ which result in a particle $z$ being emitted at the angle $\theta$ after a time $t$. The quantity $\Pi(t, b)$ is the probability that the intermediate complex characterized by an impact parameter $b$ will live a time $t$. This expression is very general and it implies a complicated folding over unknown deflection functions. It is possible to obtain reasonable results if, as done by Moretto and Sventek $[8,11]$ and by Sventek and Moretto [26], one assumes rigid rotation of the complex with a moment of inertia suitably averaged between the entrance channel and the exit channel asymmetry. In the former paper, where the calculation was performed for lighter systems, a statistical time distribution was used:

$$
\Pi(b, t)=\frac{1}{\tau} e^{-t / \tau},
$$

independent of $b$, where $\tau$ is the average lifetime of the system. An example of this calculation is shown in Figs. 32 and 33 . In the latter paper, the calculation was performed for a heavier system where the experiment suggests a much narrower time distribution which was assumed to be of the form:

$$
\Pi(t, b)=\frac{1}{N(b)} \exp -\left((t-\tau(b))^{2} / \sigma^{2}(b)\right.
$$

where $N(b)$ is a normalization constant,

$$
\begin{aligned}
& \tau(b)=\tau(0)\left(1-b / b_{\max }\right) \\
& \sigma^{2}(b)=\sigma^{2}(0)\left(1-b / b_{\max }\right) .
\end{aligned}
$$

These forms for the first and the second moment of the time distribution have a linear dependence on b suggested by trajectory calculations similar to those performed by Tsang [20]. The former and the latter expression for $\Pi(b, t)$, quite different for a heavy system like $\mathrm{Kr}+\mathrm{Au}$, give nonetheless the same result when applied to the lighter system studied in Ref. 8 because of the relatively narrow window in impact parameters leading to deep inelastic collisions in that reaction. Examples of the calculation for $\mathrm{Kr}+\mathrm{Au}$ are shown in Figs. 34 and 35. Further discussion on the agreement between experiment and theory will be given in Section IV.
SECTION II. THE MASS OR CHARGE DISTRIBUTIONS

\section{An Experimental Note}

A very powerful tool in studying heavy ion reactions is the $\Delta E$, $E$ telescope which allows one to measure the atomic number of the reaction products. This method, for its great simplicity and versatility has been widely used. In particular, in our group, we have developed a gas $\Delta E$ detector [61] that enables us to resolve individual atomic numbers up and above $Z=60$ (Fig. 2). A7l of our data presented in this paper have been taken with such a device. As a result, we have obtained $Z$ distributions, without any information on the masses. Because of the rapid equilibration between target and projectile insofar as the $\mathrm{N} / \mathrm{Z}$ ratio is concerned, we may loosely use the work mass asymmetry when we have actually measured the charge asymmetry, etc.

The Two Regimes in the Mass Distributions

The mass distributions obtained in heavy ion induced reactions can be divided in two classes. The first class includes very broad distributions, without a well defined peak in the vicinity. of the projectile and of the target. The second class includes relatively narrow distributions peaked at the target and at the projectile masses. The latter class is associated with the so called "quasi-fission" reactions, while the former is associated with the so called "deep inelastic" reactions.

Early studies seemed to suggest that very broad distributions are associated with reactions induced by light projectiles, while narrow distributions are associated with reaction induced by heavy projectiles on heavy targets. At present it appears that one can observe both kinds of distributions in the same target-projectile combination by simply varying the bombarding energy, or the ratio of the center of mass energy to the interaction barrier $E / B$. For values of $E / B$ not far from unity, the distributions are narrow and peaked in the vicinity of the target and projectile. For larger values of $E / B$, the distribution tends to become broader. In terms of the diffusion mode1, broad distributions are associated with long lifetimes, and sharply peaked distributions are associated with short lifetimes. With some 
uncertainty due to the still sketchy experimental situation, one can tentatively conclude that, for each reaction, the 7 ifetime of the intermediate complex increases with increasing $E / B$. If this is the case, one must conclude that the lifetime is a dynamical rather than a statistical quantity, and can be presumably associated with the time of contact of the two nuclei moving in and out along a radial coordinate. This point will be taken up again in the discussion about the angular distributions.

The long Lifetime Regime or the Regime of Broad Charge Distributions

The study of reactions induced by light projectiles $[1,2,5-7,27-39]$ (up to $\mathrm{Ar}$ ) on a variety of targets at fairly large energies above the interaction barrier showed the presence of two fairly well identifiable components: the quasielastic component, visible in a narrow angular range about the grazing angle and in a narrow $Z$ range above the projectile $Z$; and the relaxed component, present at all angles and essentially for a11 atomic numbers. In contrast to the narrow $Z$ distribution of the quasi-elastic component, the $Z$ distribution of the relaxed component is very broad, reminding one of fission. It was not immediately clear whether these distributions were due to compound nucleus fission, possibly enhanced by the lowering of the barrier due to angular momentum, or else a new noncompound nucleus mechanism was involved. The first possibility was favored by the nearly thermalized kinetic energy distributions associated with the relaxed or deep inelastic component of the cross section. Furthermore the general shape of the distributions $Y(Z)$ qualitatively respected the statistical prediction [27-31]:

$$
Y(Z)=K(Z) \exp -V_{Z} / T
$$

where $V_{z}$ is the potential energy of the system at the ridge point with the required mass asymmetry; and $T$ is the temperature. In other words, the cross section appeared to be high where the potential energy is low, and vice versa. This can be seen in some of the $Z$ distributions shown in Fig. 3 to Fig. 7. Furthermore, a general increase in width of the distributions, or a flattening of the slopes with increasing excitation energy, seemed to indicate a $V / T$ effect, very much statistical in nature [27-31].
However, a careful inspection showed unmistakable entrance channel effects in the $Z$ distributions, especially when comparisons of reactions expected to lead to similar compound nuclei, but with substantially different entrance channel mass asymmetries, were made [9]. For instance, in the reactions $107,109 \mathrm{Ag}+{ }^{20} \mathrm{Ne}$ (Ref. 30) and ${ }^{107,109} \mathrm{Ag}+{ }^{40} \mathrm{Ar}$ (Ref. 29) shown in Figs. 3 and 4 , the general patterns of the $Z$ distributions appear to be reversed. In the $107,109 \mathrm{Ag}+{ }^{40} \mathrm{Ar}$ reaction, one observes an increase of cross section with increasing $Z$; in the $107,109 \mathrm{Ag}+{ }^{20} \mathrm{Ne}$ reaction one observes a minimum in cross section at about $Z=15$, a sharp increase of the cross section at lower $Z$ 's and a weak increase of the cross section at higher $Z^{\prime} s$. These features seem to be definitely related to entrance channel effects. In fact in the case of $107,109 \mathrm{Ag}+{ }^{20} \mathrm{Ne}$ the cross section is largest at low $Z^{\prime} s$ in the general vicinity of $Z=10$, while, in the case of ${ }^{107,109} \mathrm{Ag}+{ }^{40} \mathrm{Ar}$, the cross section is largest at high $Z$ 's in the general vicinity of $Z=18$. These substantial changes are not expected from a compound nucleus decay, since the ridge line, controlling the statistical emission of fragments of different masses or changes, should be very similar for the two reactions.

A more plausible assumption, consistent with the observed entrance channel effects, is that the experimental $Z$ distributions are generated by a diffusion process along the mass asymmetry coordinate. If this is the case, one should be able to observe the effects of the potential energy along the mass asymmetry coordinate (ridge line) upon the diffusion process. A comparison with the ridge 1 ine potential energies and with the diffusion calculations is actually very instructive (Fig. 1). In the case of $\mathrm{Ag}+\mathrm{Ne}$, for many of the $\ell$ waves, the injection point is slightly to the left of the Businaro-Gallone mountain, leading to a rapid drift towards smaller atomic numbers, as experimentally observed. In the case of the $107,109 \mathrm{Ag}+{ }^{40} \mathrm{Ar}$ reaction, the injection point is siightly to the right of the Businaro-Gallone mountain, leading to a drift in the distribution towards larger atomic numbers, a7so as observed. For the reaction ${ }^{58} \mathrm{Ni}+{ }^{40} \mathrm{Ar}$ [5], shown in Fig. 5, a situation similar to that of ${ }^{107,109} \mathrm{Ag}+{ }^{40} \mathrm{Ar}$ arises, leading to an increasing cross section with increasing atomic number. 
In the case of the reaction ${ }^{197} \mathrm{Au}+{ }^{40} \mathrm{Ar}$ shown in Fig. 6 there is a peaking in the vicinity of the projectile at the most forward angles on a background rising with $Z$ [31]. This is consistent with the fact that the injection point is indeed to the right of the Businaro-Gallone point. However, one should not forget that more or less ordinary fission should be present.

For the reaction $107,109 \mathrm{Ag}+{ }^{86} \mathrm{Kr}$ shown in

Fig. 7, the injection point is so close to the bottom of the symmetry minimum that the $Z$ distribution at sufficiently backward angles is completely symmetric [40]. In this case, only the forward peaking angular distributions suggest that one is not dealing with compound nucleus reactions. Similarly for all other reactions mentioned above, the angular distributions are the most decisive evidence against a compound nucleus mechanism.

In conclusion, the $Z$ distributions considered so far do not easily betray their non-compound nucleus origin. The degree of relaxation along the mass asymmetry coordinate is such that only relatively weak signs of the entrance channel asymmetry are visible. The distributions are so broad that, as predicted by the fission model, phenomena reflecting the ratio $V_{z} / T$ becomes dominant. In this respect it becomes quite difficult to distinguish and resolve contributions coming from true fission from those coming from deep inelastic reactions.

The Short Lifetime Regime, or the Regime of Sharp Charge Distributions

The first reactions studied with projectiles heavier than Ar on heavy targets $[3,4,4]-45]$ immediately showed a mass distribution centered about the target and the projectile. The group who discovered the phenomenon, 3 labeled it quasi-fission, in view of the fact that the kinetic energies associated with the products were nearly thermalized or fission-like (Fig. 8). The observation of these features removes any doubt about the qualitatively different nature of these reactions from either compound nucleus or direct reactions. A more detailed study of these reactions has been performed by means of the $Z$ determination of the individual fragments. Let us consider the following reactions for sake of example: $197 \mathrm{Au}+{ }^{86} \mathrm{Kr},{ }^{159} \mathrm{~Tb}+{ }^{86} \mathrm{Kr}$, both at $620 \mathrm{MeV}$ and ${ }^{197} \mathrm{Au}+{ }^{136} \mathrm{Xe},{ }^{159} \mathrm{~Tb}+{ }^{136} \mathrm{Xe}$ both at $980 \mathrm{MeV}$. The corresponding $Z$ distributions are shown in Figs. 9-12.
Let us consider first the reaction ${ }^{197} \mathrm{Au}+{ }^{86} \mathrm{Kr}[11,46]$. The $\mathrm{Z}$ distributions shown in Fig. 9 show a various degree of peaking at the $Z$ of the projectile, depending upon the angle of measurement. Sharper $Z$ distributions are observed at intermediate angles where the angular distributions are peaking. Broader distributions are seen at more forward angles, and even broader distributions are seen at more backward angles. In the latter case it is very difficult to say where the distributions are actualiy peaking, since they are not symmetric and their maxima are so broad that the cross section is about constant over more than ten $Z$ units.

Sharp distributions, in diffusion language, are young distributions that have not had time to spread. Therefore, moving from forward to backward angle, we have the sequence: middle age, young, old distributions. We have commented elsewhere $[11,46]$ that this is strongly suggestive of a lifetime decreasing with increasing angular momentum.

For the same velocity $v_{0}$ at the interaction radius, small impact parameters have a large radial velocity (which may mean large radial interpenetration and long lifetime) and a slow angular velocity, while the largest impact parameters have a small radial velocity and thus a short lifetime, and a large angular velocity. Therefore, it seems possible to associate the backward angle distributions with large impact parameters, the intermediate angle distributions with small impact parameters and the forward angle distributions with intermediate impact parameters.

This association is also justified in terms of angular velocity. Small impact parameter systems live long but rotate slowly and cannot reach very forward angies. Large impact parameter systems rotate much faster, but decay so soon that they cannot rotate too forward. The intermediate impact parameters have optimal angular velocity and lifetime to reach the most forward angles.

In support of what has been said above, it can be observed that the young distributions are not very well relaxed in kinetic energy, while the middle age and the old distributions have progressively more relaxed kinetic energy distributions. Comments along the same line have been made also by wolf and Roche [47]. In the ${ }^{159} \mathrm{~Tb}+{ }^{86} \mathrm{Kr}$ reaction (Fig. 10 ), the same pattern can be observed [48]. The general 
picture, however, is that of a more extensive relaxation. In particular, the most backward distributions are not at all peaked in the neighborhood of the projectile, nor are the intermediate angle $Z$ distributions if their higher energy component (quasi-elastic) is removed. In both reactions one would like to see a drift of the mass distribution peak towards symmetry. However, while there is an excess cross section at larger Z's, it is hard to see a well defined peak in the backward angle distributions. In many respects the $Z$ distributions at backward angles, expecially for ${ }^{159} \mathrm{~Tb}+{ }^{86} \mathrm{Kr}$, begin to resemble those observed in the ${ }^{107,109} \mathrm{Ag}+{ }^{86} \mathrm{Kr}$ reaction [40], illus trated above.

In the reactions ${ }^{197} \mathrm{Au}+{ }^{136} \mathrm{Xe}$ [49] and ${ }^{159} \mathrm{~Tb}+{ }^{136} \mathrm{Xe}$ [50] at $979 \mathrm{MeV}$ (Figs. 11 and 12), the short decay time features are even more enhanced. The peaking at the projectile seems to persist over a broader angular range than in the previous reactions.

In the ${ }^{197} \mathrm{Au}+{ }^{136} \mathrm{Xe}$ reaction, a strong fission component is observed. It can be separated from the deep inelastic component because the kinetic energy spectrum shows two peaks. This component, arises from the fission of the quasi $\mathrm{Au}$ fragment. The fission of the quasi target is essentialiy absent in the reaction ${ }^{159} \mathrm{~Tb}+{ }^{136} \mathrm{Xe}$ because of the much higher fission barriers involved.

As a final example of mass distributions we consider those arising from light target-projectile combinations studied in the previous subsection, but at much smaller values of $E / B$.

The reaction ${ }^{197} \mathrm{Au}+{ }^{40} \mathrm{Ar}$, which, already at $288 \mathrm{MeV}$ bombarding energy shows a peak in the mass distribution in the vicinity of the projectile, and other quasi-fission features [31] (Figs. 6 and 23), shows at $220 \mathrm{MeV}$ a more dramatic quasi-fission pattern in the mass distribution [51]. Similarly the reaction ${ }^{63} \mathrm{Cu}+{ }^{93} \mathrm{Nb}$ at $280 \mathrm{MeV}$ shows a mass distribution centered about the projectile and the target [52].

Even the reaction $107,109 \mathrm{Ag}+{ }^{40} \mathrm{Ar}$ changes its charge distribution [53] when the energy is lowered at $170 \mathrm{MeV}$, showing two peaks. A first sharp peak is centered at the projectile and a broader peak seems to be centered at symmetry (see Fig. 13). This represents the evidence indicating that the mass distributions should be classified in terms of the ratio $E / B$ rather than in terms of the target-projectile combinations.

Dependence of the $Z$ Distribution Upon Energy Dissipation

In many reactions, especially in those of the quasi-fission type, the relaxed and the quasielastic components are, at times, bridged by a partially relaxed component. It is then desirable to find out the dependence of the charge or mass distribution on the degree of damping.

The mass distributions, when observed for bins of decreasing fragment kinetic energy $[11,54,55]$ start out very narrow and become progressively broader. Their shape is approximately Gaussian and the centroid at times seems to drift with decreasing kinetic energies, at other times it seems to remain fixed at the $Z$ of the projectile.

Examples of such distributions are given in Fig. 14 and Fig. 15. In certain cases these distributions are given for a fixed lab. angle [11], in other cases, like in Fig. 14 and Fig. 15, the distributions are integrated over angle. The $Z$ distributions shown in Figs. 14 and 15 have been obtained by defining the energy bins by means of $E_{\text {Fragment }}$ vs. $Z$ lines parallel to the experimenta, $E_{\text {Fragment }}$ vs. $Z$ line corresponding to the relaxed kinetic energy centroids as determined from measurements at backward angle. This fancy procedure has the advantage of defining the energy bins for constant energies above the completely relaxed energy line.

The qualitative meaning of these distributions is clear. At small degrees of energy relaxation, one observes narrow $Z$ distributions, while at greater degrees of energy damping, the $Z$ distribution become substantially larger. This shows that the diffusion in mass asymmetry occurs also before complete energy relaxation, which is not too unexpected.

The quantitative use of these data is not as simple as it may appear. One for instance could use the equations derived in Section I in order to relate the widths of the mass distributions to their centroids, or to relate the widths of the mass distributions to the energy loss. Unfortunately, these data, either taken at fixed angle or integrated over angle contain contributions from different $\&$ waves which may vary from bin to bin in a complicated way. And, since the diffusion properties are different from each $\ell$ wave, it 
seems reasonable to conclude that the analysis of these data without unfolding the $\ell$ distribution can yield only qualitative, or at best semiquantitative results.

This point has been touched in Section I and will be discussed again in Section IV.

SECTION III. THE ANGULAR DISTRIBUTIONS

Lifetime and Rotational Period, or the Two Angular Distribution Regimes

As in the case of the charge distributions discussed in the previous section, two kinds of angular distributions are observed in deep inelastic reactions. Forward peaked angular distributions are observed, frequentiy, but not necessarily associated with broad $Z$ distributions, and side-peaked angular distributions are observed usually associated with narrow $Z$ distributions, peaked at the $Z$ of the projectile.

As will be seen later on in this section, there is a continuous evolution from one kind of angular distribution to the other. The physical quantity which determines the prevailing angular distribution regime is the ratio between the lifetime of the complex and its mean rotational period. If this ratio is sma11, the system does not have a chance to rotate enough to reach $0^{\circ}$, and consequently the products are emitted at wide angles, on the same side of the impact. If this ratio is large and approaches one, the system rotates past $0^{\circ}$ decaying in the meantime. This generates a forward-peaked angular distribution. If this ratio is very large, much larger than one, the system has a chance to rotate several times before it decays and, if the lifetime distribution is sufficiently broad, the angular distribution will become symmetric about $90^{\circ}$ and will tend, for large angular momenta to the $1 / \sin \theta$ iimit.

In order to evaluate such a ratio, one needs a rather detailed and sophisticated model. However, it has been empirically observed that the ratio $E / B$ is a good predictor of the angular distribution regimes. At $E / B$ values below 1.6, one observes side peaking, while for values of $E / B$ above 1.6, one observes a forward peaking.

The possible reason why $E / B$ is a good empirical parameter can be seen as follows. Let us consider the quantity:

$$
\frac{E}{B}-1=\frac{E-B}{B}
$$

Provided that the Coulomb barrier is close to the interaction barrier, the above quantity can be written as follows:

$$
\begin{aligned}
\frac{E-B}{B} \simeq \frac{\mu v_{0}^{2} R}{2 Z_{1} Z_{2}}=\frac{1}{4}\left[\frac{2 \mu v_{0} R^{2}}{Z_{1} Z_{2}}\right]\left[\begin{array}{c}
v_{0} \\
R
\end{array}\right]= \\
\frac{1}{2}\left[2 \mu \frac{v_{0}}{\sqrt{2}} \frac{R^{2}}{Z_{1} Z_{2}}\right]\left[\frac{v_{0}}{\sqrt{2}} \frac{1}{R}\right]
\end{aligned}
$$

where $\mu$ is the reduced mass of the target-projectile at the interaction radius $R ; v_{0}$ is the center of mass velocity at the same radius; and $z_{1}, z_{2}$ are the target and projectile atomic numbers.

Let us now consider an impact parameter such that the radial and tangentile velocities are the same. At the interaction radius these velocities are $\frac{v_{0}}{\sqrt{2}}$. Then the first square bracket in the last expression represents the time it takes the system, subject to a force equal to the Coulomb force at the interaction radius, to move radially, in and out. Insofar as viscosity is neglected (which may be dangerous); insofar as the Coulomb force is representative of the forces at the interaction radius (which is not as bad as it sounds because the centrifugal force at sufficiently larger $\&$ values may partly compensate the nuclear force); and for a moderate value of $v_{0}$, the first square bracket can be identified with the lifetime:

$$
\tau_{\text {T ifetime }}=2 \frac{\mu v}{c} \simeq 2 \frac{v_{0}}{\sqrt{2}} \mu \frac{R^{2}}{Z_{1} z_{2}}=\frac{\sqrt{2} h l_{\text {max }}}{B},
$$

where $\underset{\sim}{v}$ is the velocity; $c$ is the force; $\ell_{\max }$ is the maximum $l$ wave associated with the reaction.

The second square bracket is just the angular velocity (assuming no sticking and no dissipation).

The product of the two brackets is the angle of rotation of the complex, prior to decay:

$$
\theta_{\text {Rot }}=2\left(\frac{E}{B}-1\right)
$$

A rotation of 1 radian may be a good criterion for discriminating between orbiting past $0^{\circ}$ and side decay. We then obtain:

$$
1=2\left(\frac{E}{B}-1\right), \text { or } \frac{E}{B} \simeq 1.5
$$

In conclusion $2\left(\frac{E}{B}-1\right)$ represents the product of a lifetime of some sort and of an angular velocity. 
The rotation of a radian is obtained with $E / B=1.5$. For those who do not like this a posteriori justification, Table I shows that indeed for $E / B$ around 1.6 or lower, the side peaking in the angular distribution is well established while for values larger than 1.6 the angular distributions are completely forward peaked.

The Regime of Long Relative Lifetimes $(E / B>1.6)$

As it was seen in the previous section, reactions induced by fairly light projectiles (up to $\mathrm{Ar}$ ) at moderately high energies, are characterized by broad mass distributions. The nearly thermalized kinetic energy spectra associated with such products, together with the broad $Z$ distributions are very reminiscent of compound nucleus fission. On the other hand, various features in the $Z$ distribution suggested a noncompound nucleus mechanism.
However, remarkable features observed in the angular distributions $[1,2,5-7,27-39]$, like forward peaking in excess of $1 / \sin \theta$ and its dependence upon the $Z$ difference between the actual fragment and the projectile are crucial in ruling out compound nucleus reactions. It is worth repeating that this forward peaking is associated with the relaxed component of the cross section. While in many cases the forward peaking is associated with slightiy higher kinetic energies, especially at angles smaller than the grazing angle, still such increases are minimal when compared with the bombarding energy (Fig. 23). The slight increase in kinetic energy with angle appears to be associated with an increase in impact parameter leading to a decreased degree of energy dissipation.

An overall picture of the features associated with the angular distribution as a function of the

Table I.

\begin{tabular}{|c|c|c|c|c|}
\hline Reaction & $E_{\mathrm{Lab}}(\mathrm{MeV})$ & $\mathrm{E}_{\mathrm{CM}}(\mathrm{MeV})$ & $\mathrm{B}(\mathrm{MeV})$ & $E / B$ \\
\hline \multirow[t]{3}{*}{$107,109 \mathrm{Ag}+{ }^{40} \mathrm{Ar}$} & 340.0 & 248.0 & 101.0 & 2.46 \\
\hline & 288.0 & 210.0 & 101.0 & 2.08 \\
\hline & 170.0 & 124.0 & 101.0 & $* 7.22$ \\
\hline \multirow[t]{3}{*}{${ }^{197} \mathrm{Au}+{ }^{40} \mathrm{Ar}$} & 340.0 & 283.0 & 154.0 & 1.84 \\
\hline & 288.0 & 239.0 & 154.0 & $* ? 7.55$ \\
\hline & 220.0 & 182.0 & 754.0 & $* 1.19$ \\
\hline${ }^{83} \mathrm{Nb}+{ }^{63} \mathrm{Cu}$ & 280.0 & 167.0 & 138.0 & $* 7.21$ \\
\hline \multirow[t]{2}{*}{${ }^{197} \mathrm{Au}+{ }^{63} \mathrm{Cu}$} & 443.0 & 336.0 & 235.0 & $\star 1.43$ \\
\hline & 365.0 & 276.0 & 235.0 & *1.17 \\
\hline $107,109 \mathrm{Ag}+{ }^{86} \mathrm{Kr}$ & 620.0 & 339.0 & 184.0 & 1.84 \\
\hline${ }^{159} \mathrm{~Tb}+{ }^{84} \mathrm{Kr}$ & 620.0 & 402.0 & 240.0 & 1.67 \\
\hline${ }^{181} \mathrm{Ta}+{ }^{86} \mathrm{Kr}$ & 620.0 & 420.0 & 264.0 & $\star 1.59$ \\
\hline${ }^{197} \mathrm{Au}+{ }^{86} \mathrm{Kr}$ & 620.0 & 431.0 & 282.0 & $* 1.53$ \\
\hline${ }^{159} \mathrm{~Tb}+{ }^{136} \mathrm{Xe}$ & 980.0 & 528.0 & 339.0 & $\star 1.56$ \\
\hline${ }^{197} \mathrm{Au}+{ }^{136} \mathrm{Xe}$ & 980.0 & 579.0 & 399.0 & $* 1.45$ \\
\hline
\end{tabular}


$Z$ of the fragment can be obtained from Fig. 16 to Fig. 21. In all of these reactions the most remarkable feature is the excess forward peaking in the center of mass angular distributions, especialiy in the vicinity of the projectile.

The forward peaking implies the existence of an intermediate complex with a lifetime shorter than the mean rotational period. It also suggests that such an intermediate complex retains in its shape a memory of the initial target-projectile combination. This is an important observation: a completely randomized shape, even if associated with a very short 1 ifetime, would not lead to a backward-forward asymmetry. A further observation is that the kinetic energy dissipation occurs on a time scale shorter than both the rotational period and the mean lifetime.

The extend of rotation prior to the decay of the intermediate complex can be established approximately by the following observations: the forward peaking implies orbiting past $0^{\circ}$, while the presence of fairly large cross sections, and occasionally even of a minor peaking in the backward direction suggests that, at least at times, orbiting extends to almost one complete rotation, as far back as $180^{\circ}$.

By far the most important feature, observed in all these reactions, is the dependence of the angular distribution upon the difference in $Z$ between the observed fragment and the projectile. More specifically, the forward peaking is more pronounced in the vicinity of the projectile, and fades away toward the $1 / \sin \theta 1$ imit for fragments with Z's far removed from the projectile.

This phenomenon finds its qualitative explanation $[8,9,11]$ in an increasing time lag associated with the population of configurations farther removed in mass asymmetry from the injection asymmetry. Configurations with fragments close in $Z$ to the projectile are quickly populated and can quickly decay, thus generating a substantial forward peaking. Fragments farther removed from the projectile are populated on a larger time scale and decay over a longer time period, thus generating angular distributions more symmetric about $90^{\circ}$. It was such a peculiar combination of kinetic energy thermalization, associated with a time evolution along the mass asymmetry coordinate that led us to postulate the existence of an intermediate complex, nearly equilibrated in all the collective degrees of freedom and evolving along the mass asymmetry mode by means of the diffusion mechanism $[8,9,17]$.

The apparent effect of the ridge-line potential upon the time evolution of the system also seems to suggest a diffusion process. For instance, the disappearence of the excess forward peaking as one moves away in $Z$ from the projectile, appears to be asymmetric at times. In $14 \mathrm{~N}+107,109 \mathrm{Ag}$ reaction [28], and also in $20 \mathrm{Ne}+107,109 \mathrm{Ag}$ reaction [30] shown in Fig. 16, the forward peaking is more pronounced and persistent for fragments lower in $Z$ than the projectile, while, for higher $Z$ fragments, the excess forward peaking rapidly disappears. A check of the potential energy vs. mass asymmetry shows that for both of these reactions, the injection point lies more or less to the left of the Businaro-Gallone mountain (Fig. 1). Consequent1y, the potential energy appears to drive the diffusion process rapidly to the left and slowly to the right of the injection point. Configurations associated with fragments lower in $Z$ than the projectile are quickly populated and they can quickly decay with the resulting sharp forward peaking. Instead, the fragments higher in $Z$ than the projectile are populated on a slower time scale and consequently the excess forward peaking rapidly disappears. An inversion of these phenomena can be observed in the reaction ${ }^{197} \mathrm{Au}+{ }^{40} \mathrm{Ar}$ at both 288 and $340 \mathrm{MeV}$ bombarding energy [31] (Fig. 17). In this case, the injection point is to the right of the Businaro-Gallone mountain and the potential drives the diffusion towards symmetry. The experimental data shows that, contrary to the $107,109 \mathrm{Ag}+{ }^{20} \mathrm{Ne}$ case, the excess forward peaking is retained from $Z=18$ to about $Z=29$ or 11 atomic numbers above the projectile. A distance of only 4 to 5 atomic numbers was sufficient to reduce the angular distributions to the $1 / \sin \theta$ form in the case of ${ }^{20} \mathrm{Ne}+{ }^{107,109} \mathrm{Ag}$.

An intermediate situation occurs for the reactions $107,109 \mathrm{Ag}+40 \mathrm{Ar}$ (Fig. 18) [29] and ${ }^{58} \mathrm{Ni}+{ }^{40} \mathrm{Ar}$ (Fig. 19) [5]. In both cases the injection point is slightly to the right of the Businaro-Gallone mountain. This hampers, to some extent, the diffusion to the left of the injection point. On the other hand, once over the hump, the systems can rapidly diffuse downhill toward the lowest Z's. This may explain why the forward peaking is not quite a pronounced as in the previous 
reactions but it extends over a remarkably great $z$ interval.

The completely symmetric reaction of ${ }^{40} \mathrm{Ca}+{ }^{40} \mathrm{Ca}$ [34] also shows the progressive disappearance of the excess forward peaking in the anguiar distributions as one moves away from the injection point (Fig. 20).

The magnitude of the forward peaking in a given reaction appears to be determined by the bombarding energy. A sharper forward peaking is observed at lower bombarding energies. See for instance Figs. 16 and 17 . This effect is certainly related to the obvious decrease of the parameter $E / B$ and finds its, qualitative explanation in the decrease of the rotational frequency and in the lifetime as well. A further decrease of $E / B$ does in fact lead to a side peaking in the angular distributions.

It is worth noticing that even the reaction $107,109 \mathrm{Ag}+{ }^{86} \mathrm{Kr}$, at $620 \mathrm{MeV}$ bombarding energy [40], falls in th category of long relative 1 ifetime $(E / B=1.84)$. The angular distributions (Fig. 21) clearly manifest this limit in their complete lack of side peaking and in a persistent forward peaking, visible for most of the fragments.

Let us consider the reactions $107,109 \mathrm{Ag}+{ }^{86} \mathrm{Kr}$ $(E / B=1.84)[40]$ and ${ }^{797} \mathrm{Au}+{ }^{40} \mathrm{Ar}(\mathrm{E} / \mathrm{B}=1.55)$ [37] in more detail because they illustrate the transition from a long lifetime relative to the rotational period, to a short relative lifetime. In Fig. 22 the Wilczynski plots for some of the fragments with $Z$ close to the projectile are shown for $107,109 \mathrm{Ag}+{ }^{86} \mathrm{Kr}$. The patterns are typical of an orbiting regime with a well developed relaxed component. Quite a different (and puzzling) picture is presented by the ${ }^{197} \mathrm{Au}+{ }^{40} \mathrm{Ar}$ reaction (Fig. 23). For $Z>Z_{\text {proj }}$ the patterns correspond to an extensive orbiting regime. The kinetic energies rapidly become completely relaxed as $Z$ increases and the angular distributions are forward peaked. Instead, for $z<z_{\text {proj }}$ the kinetic energies do not relax completely, the evidence of orbiting is reduced and sizable side peaking is observed in the angular distributions. These features tend to anticipate the features observed in the so called quasi-fission process. It is not clear as yet why in the reaction ${ }^{197} \mathrm{Au}+{ }^{40} \mathrm{Ar}$ such dramatic difference should exist between the low Z's and the high Z's.
The Regime of Short Relative Lifetimes

The earlier measurements of angular distributions for quasi-fission reactions were gross angular distributions, which included all the products at once $[3,4,41-45]$. These angular distributions are side peaked, the peak appearing in the neighborhood of the grazing angle.

Incidentally, the association of the rainbow angle with the grazing angle is a bit mystifying. Although there is an undeniable experimental correlation between the two, one should not take such a correlation to be evidence of a strict genetic relationship. In fact, the tangential velocity as well as the radial velocity at distances close to the interaction radii should be substantially reduced, as shown by the low exit channel kinetic energies. This implies that the correlation between grazing angle and rainbow angle is more complicated than that observed for the quasi-elastic reactions. The simplest conclusion one can draw from the side peaking is that the lifetime of the complex is, on the average, so short as compared to the rotational period, that the rotation does not extend past $0^{\circ}$.

The remarkable features of the side peaked angular distributions, together with the mass distributions peaking at about the projectile mass, may lead to the speculation that quasifission is a process distinct from the deep inelastic processes which are instead characterized by broad mass distributions and by forward peaked angular distributions. However, we have shown experimentally that the two processes are in fact one and the same, the differences being simply related to the differences in lifetime and rotational period.

Let us consider the mass (or $Z$ ) distribution. If the spread in mass (or Z) is due to the diffusion mechanism, the lifetime should increase with the difference between the $Z$ of the observed fragment and that of the projectile. If the increase in lifetime is sufficiently large, one may expect the onset of orbiting beyond $0^{\circ}$, which causes the angular distribution to evolve from side peaked to forward peaked.

This is exactly what is observed in the experiment of ${ }^{197} \mathrm{Au}+620 \mathrm{MeV}{ }^{86} \mathrm{Kr}$ where we studied the angular distributions as a function of atomic number of the fragments [46]. Figure 24 shows a 
sharp side peak at $Z=36$. As one moves towards either lower or higher $Z$ 's, one observes a movement of the side peak towards $0^{\circ}$, a simultaneous increase of the cross section at $0^{\circ}$ and the evolution of the side peak into a shoulder first, and into a forward peak later for Z's sufficiently removed for that of the projectile.

The evolution from side peaking to forward peaking can be better appreciated in the Wilczynski plots shown in Fig. 25. As expected, this evolution of the angular distribution is nearly symmetric about the projectile and strongly supports the assumption of a diffusion mechanism along the mass asymmetry degree of freedom. At the same time, a continuous connection is established between forward peaked angular distributions and side peaked angular distributions, showing the essential identity of quasi-fission and the other deep inelastic processes. Similar features have been observed in the angular distributions for various mass windows in the reaction ${ }^{197} \mathrm{Au}+365 \mathrm{MeV}$ ${ }^{63} \mathrm{Cu}$ studied by Péter et a1. [42].

The overall evolution from side peaking to forward peaking can be controlled by simply altering the E/B ratio. Good examples of this feature are the reactions ${ }^{197} \mathrm{Au}+{ }^{63} \mathrm{Cu}$ at $365 \mathrm{MeV}$ $(E / B=1.17)$ and at $443 \mathrm{MeV}(E / B=1.43)$ studied by Tamain et a1. [56] (Fig. 26) where the broadening of the side peak and its shift towards smaller angle suggests the onset of orbiting. Orbiting is also completely evident in the reaction $165_{\mathrm{Ho}}+{ }^{84} \mathrm{Kr}$ at $714 \mathrm{MeV}(\mathrm{E} / \mathrm{B}=1.89)$ studied by Birkelund et al. [57] where even the gross kinetic energy spectra show the double peaking so typical of reactions induced by lighter projectiles.

The angular distribution shown in Fig. 27 for ${ }^{159} \mathrm{~Tb}+{ }^{86} \mathrm{Kr}$ at $620 \mathrm{MeV}(E / \mathrm{B}=1.67)$ [48] portray a very smal1 amount of side peaking in the immediate vicinity of the projectile, mostly due to incompletely relaxed events. A predominance of the forward peaking (or orbiting) is observed for all the remaining 2 's. An extreme orbiting regime can be seen again in the reaction $107,109 \mathrm{Ag}+{ }^{86} \mathrm{Kr}$ at $620 \mathrm{MeV}$ [40] $(\mathrm{E} / \mathrm{B}=1.84)$, described above (Figs. 21 and 22).

At the other extreme, the reaction ${ }^{197} \mathrm{Au}+{ }^{136} \mathrm{Xe}$ at $979 \mathrm{MeV}(\mathrm{E} / \mathrm{B}=1.45)$ can be considered [49]. In Figs. 28 and 29, the angular distributions and the Wilczynski plots are shown as a function of $Z$. The side peaking dominates the angular distributions even at $Z$ 's very far removed from the projectile, al though for very low Z's an incipient orbiting is visible in the rising forward angle component of the angular distribution.

A more detailed comparison between the last reaction and the reaction ${ }^{197} \mathrm{Au}+{ }^{86} \mathrm{Kr}$ at $620 \mathrm{MeV}$ seems to show interesting effects, possibly due to an enhanced deformation of the touching fragments in the former system. The first indication is in the average kinetic energies as a function of $Z$, which, when compared to the Coulomb energies of two touching spheres, are lower for the ${ }^{197} \mathrm{Au}+{ }^{136} \mathrm{Xe}$ reaction. This despite the fact that the total charge is larger in the ${ }^{197} \mathrm{Au}+{ }^{136} \mathrm{Xe}$ case and that the mean rotational energies evaluated for the spherical target and projectile in contact are $63 \mathrm{MeV}$ for ${ }^{197} \mathrm{Au}+{ }^{136} \mathrm{Xe}$ and $49 \mathrm{MeV}$ for ${ }^{197} \mathrm{Au}+{ }^{86} \mathrm{Kr}$. Furthermore, the calculated mean rotational periods, for the same spherical configurations, are very similar to each other: $0.94 \times 10^{-20} \mathrm{sec}$ and $0.90 \times 10^{20} \mathrm{sec}$ respectively. And, since the $Z$ distributions are quite simitar in width; thus indicating a comparable lifetime, one should expect a comparable side peaking. However, the side peaking is much more pronounced in the $\mathrm{Xe}$ than in the $\mathrm{Kr}$ induced reaction. This may indicate a decrease in the angular velocity brought about by an elongation of the ${ }^{197} \mathrm{Au}+{ }^{136} \mathrm{Xe}$ system.

Much more comparable with ${ }^{197} \mathrm{Au}+{ }^{86} \mathrm{Kr}$ is the reaction ${ }^{159} \mathrm{~Tb}+{ }^{136} \mathrm{Xe}$ where the extent of side peaking and its persistence with $Z$ is very similar to that observed in ${ }^{197} \mathrm{Au}+{ }^{86} \mathrm{Kr}$ (Fig. 30).

Angular Distributions as a Function of Fragment Kinetic Energy

In many cases, but expecially in the cases in which one observes side peaked angular distributions, like in the $\mathrm{Kr}$ and $\mathrm{Xe}$ induced reactions, there is not a compiete separation between the quasi-elastic and the relaxed component. Furthermore, the so called relaxed component is at times very broad in kinetic energy. This suggests a study of the angular distributions as a function of kinetic energy. The examples shown in Fig. 31 for the ${ }^{197} \mathrm{Au}+{ }^{86} \mathrm{Kr}$ at $620 \mathrm{MeV}$ [46] indicate that a strong side peaking in the angular distributions is observed at the higher. kinetic energies and for Z's close to the projectile. As the kinetic energy decreases, the side peaks become broader and eventually degenerates into a forward peaking (see also Ref. 58). This variation in the angular 
distributions within the "relaxed" peak is not well understood. A possible connection can be made with the rate of energy dissipation, which has been studied by Birkelund, et a1. [55]. As shown in Fig. 38, the initial stage of energy dissipation is fast, while the latter stage appears to be very slow. It seems conceivable that the lowest kinetic energy products are associated with larger decay times and manifest their long life in the forward peaking of their angular distribution.

SECTION IV. DIFFUSION MODEL RESULTS, OIFFUSION CONSTANTS AND FOKKER-PLANCK EQUATION COEFFICIENTS

Moretto and Sventek [8] tried to fit the experimental cross sections and angular distributions for the reaction $107,109 \mathrm{Ag}+{ }^{40} \mathrm{Ar}$ at $288 \mathrm{MeV}$ bombarding energy. The results, shown in Figs. 32 and 33 indicate that the angular distribution patterns as a function of the $Z$ of the fragments are well reproduced. In particular, the disappearance of the excess forward peaking with increasing distance from the projectile is obtained. A direct comparison with the data shows that the calculation reproduces the experiment to within $30 \%$. In the calculation, the assumption was made that a11 of the relaxed component of the cross section is associated with the decay of the intermediate complex and that compound nucleus fission is absent. The arguments justifying this hypothesis can be found in Ref. 8. The parameters obtained from the fitting are:

$$
\text { diffusion constant } k=0.5 \times 10^{20}
$$

$$
\text { charge units } \sec ^{-1} \mathrm{fm}^{-2}
$$

mean decay time $\tau=2.5 \times 10^{-21} \mathrm{sec}$

In another paper Sventek and Moretto [26] tried to reproduce the angular distributions for ${ }^{197} \mathrm{Au}+{ }^{86} \mathrm{Kr}$ at $620 \mathrm{MeV}$ bombarding energy using the same formalism. The results of their work can be seen in Fig. 34. The angular distribution patterns are qualitatively reproduced, in particular the disappearance of the side peaking in the angular distributions with increasing distance from the projectile. This indicates that the diffusion process can satisfactorily explain the increasing time delay associated with increasing distance from the projectile which is responsible for the transformation of the side peaked angular distributions into a forward peaked angular distributions. The comparison between experimental and calculated $Z$ distributions as a function of angle (Fig. 35) shows that the correct pattern of sharper distributions at intermediate angles, and broader distribution at more forward and backward angles, is also reproduced. However, on the whole, the calculated distributions are a bit too narrow and too much displaced towards large Z's than the data. This is certainiy due to a ridge line which is too steep, and which tends to introduce an excessive drift. The parameters obtained are:

$$
\begin{aligned}
& \kappa=0.25 \times 10^{21} \text { charge units } \mathrm{sec}^{-1} \mathrm{fm}^{-2} \\
& \tau(0)=3.5 \times 10^{-21} \mathrm{sec}
\end{aligned}
$$

Here $\tau(0)$ is the 1 ifetime at $\ell=0$ (see Eqs. (31) and (32)) and should not be compared directly with the $\ell$ independent $\tau$ of the previous example (see Eq. (30)). The quantity $\tau(0)$ is fixed quite accurately by the position of the side peak. It follows that the value of $k$ assumed in the calculation is in fact too sma11, for it gives too narrow widths. By adjusting the slope of the potential, it is possible to obtain the correct dirft and spread for a value of $k=0.5 \times 10^{-21}$ charge units $\mathrm{sec}^{-1} \mathrm{fm}^{-2}$. Unfortunately, we have not determined withcertainty as yet which shape of the intermediate complex would reproduce the necessary slope. The important thing, however, is that the quantity $K$ in both experiments seems to be around $\kappa=0.5 \times 10^{21}$ charge units $\sec ^{-1} \mathrm{fm}^{-2}$. From it we can calculate the approximate drift and spread coefficients associated with the Fokker-Planck Equation. However, we must point out very strongly that, while $k$ is essentially independent of both excitation energies and angular momentum, the Fokker-Planck coefficients $\mu_{1}$ and $\mu_{2}$ are dependent on both, as well as on the derivative of the potential energy with respect to $Z$. Therefore, in principle, for an actual reaction, one should compute $\mu_{1}$ and $\mu_{2}$ for each $\ell$ wave and for each asymmetry. Since values of $\mu_{1}$ and $\mu_{2}$ obtained for an unknown distribution of $l$ waves have been reported in 1iterature, we shall calculate the same coefficients for some reasonable average $\ell$ wave, for sake of comparison.

The results are the following: For $\mathrm{Au}+620 \mathrm{MeV}{ }^{86} \mathrm{Kr}$ (using $\mathrm{K}=0.5 \times 10^{21}$ charge units $\mathrm{sec}^{-1} \mathrm{fm}^{-2}$ : 
$\mu_{1}=3.0 \times 10^{21}$ charge units $\mathrm{sec}^{-1}$
$\mu_{2}=0.66 \times 10^{22}$ (charge units) $^{2} \mathrm{sec}^{-1}$
$\mu_{2} / \mu_{1}=2.23$$\left\{\begin{array}{l}\ell=106 \mathrm{~h} \\ V^{\prime}=1.23 \mathrm{MeV} / \\ \text { charge unit } \\ \mathrm{T}=1.47 \mathrm{MeV}\end{array}\right.$

For $\mathrm{Ag}+{ }^{40} \mathrm{Ar}$ at $288 \mathrm{MeV}$ :

$\mu_{1}=0.79 \times 10^{21}$ charge units $\sec ^{-1} \mid \ell=99$

$\mu_{2}=5.1 \times 10^{21}$ (charge units) $^{2} \mathrm{sec}^{-1}\left\{\begin{array}{l}l=99 \\ \mu_{2} / \mu_{1}=6.5\end{array} \quad \begin{array}{l}0.6 \mathrm{MeV} / \\ \text { charge unit } \\ \mathrm{T}=1.94 \mathrm{MeV}\end{array}\right.$

Nörenberg $[12,13]$ has analyzed the ${ }^{232} \mathrm{Th}+{ }^{40} \mathrm{Ar}$ reaction at $295 \mathrm{MeV}$ by establishing a linear relationship between both the centroid and the second moment of the $Z$ distribution with angle $\theta$ (see Eqs. (19) and (20)) and by transforming the angle into time by means of the relation given by Eq. (22). The results are:

$$
\begin{aligned}
& \mu_{1}=1.0 \times 10^{21} \text { charge units } \mathrm{sec}^{-1} \\
& \mu_{2}=2.0 \times 10^{22} \text { (charge units) } \mathrm{sec}^{-1} .
\end{aligned}
$$

otice that here the ratio is:

$$
\mu_{2} / \mu_{1}=20 \text {. }
$$

Since the ratio, with great generality, is given by $\frac{2 T}{V_{z}^{\prime}}$ this result appears rather strange since $V_{z}^{\prime}$ is approximately $1.0 \mathrm{MeV}$ and $\mathrm{T}$ somewhere between $?$ and $3 \mathrm{MeV}$. A similar analysis erformed by Wolf et a 1. $[47,59]$ on ${ }^{165} \mathrm{Ho}+{ }^{84} \mathrm{Kr}$ at $714 \mathrm{MeV}$ and on other reactions gives

$$
\mu_{2} \simeq 4.0 \times 10^{22} \text { (charge units) }{ }^{2} \mathrm{sec}^{-1} .
$$

These coefficients are substantially larger than those obtained by us. Consequently, al though the beautiful linear relationship between $\sigma_{z}^{2}$ and $\theta$ given by the above authors $[47,59]$ in Fig. 36 is rather impressive, we feel that the observed widths may be broadened by the \& distribution and may lead to excessively large $\mu_{2}$ coefficients when used as such in the Fokker-Planck Equation. As an example we report a calculation of $\sigma_{z}^{2}$ vs. $Z-z_{0}$ for various $\ell$ waves and for the weighted average of the distributions for the reaction ${ }^{197} \mathrm{Au}+{ }^{86} \mathrm{Kr}$ (Fig. 37). It is obvious that there is a great difference from one $\ell$ value to another and it is not clear what meaning can be assigned to the quantities obtained from the weighted average of the distributions.

The analysis by $\mathrm{Ngô}$ et al. [25] of the data for the reaction ${ }^{197} \mathrm{Au}+{ }^{63} \mathrm{Cu}$ at $443 \mathrm{MeV}$ and at $365 \mathrm{MeV}$ is less ambitious but may suffer from the same difficulties. They plot $\sigma_{z}^{2}$ vs. $z-z_{0}$ and from the resulting linear plot they obtain:

$$
\begin{aligned}
& \mu_{2} / \mu_{1}=6.12 \text { at } 443 \mathrm{MeV} \\
& \mu_{2} / \mu_{1}=3.06 \text { at } 365 \mathrm{MeV}
\end{aligned}
$$

in almost exact agreement with the equation:

$$
\mu_{2} / \mu_{1}=\frac{2 T}{V_{Z}^{\prime}}
$$

In this equation $V^{\prime}=0.7 \mathrm{MeV}$ (charge unit) ${ }^{-1}$ as calculated for $\ell=0$; and $T$ is calculated using $a=\frac{A}{15}$ and the excitation energy of the intermediate complex for $l=0$. The latter assumption we judge unsafe because it leads to an overestimate of the temperatures and thus of the widths, which experimentally are broadened by the average over the $\ell$ distribution. Another possible difficulty with this work regards the derivation of the means and the widths from data which deviate severely from a Gaussian shape required by the theory being applied.

of great interest is the attempt to use the proportionality between $\sigma_{z}^{2}$ and time:

$$
\sigma_{z}^{2}=\mu_{2} t
$$

by Birkelund et a $1 .[55,60]$ in order to establish the rate of energy dissipation (Fig. 38). It can be seen that the energy dissipation occurs in two stages, a fast stage which removes about $80 \%$ of the energy, and a slow stage which removes the remaining energy. During the first stage, very little diffusion occurs along the mass asymmetry mode, while substantial mass diffusion occurs during the second stage.

One must exercise some caution in drawing quantitative conclusions from the use of the experimental $\sigma_{z}^{2}$. From the previous discussion it appears that the use of $\sigma_{z}^{2}$ obtained from the experimental distributions may in fact lead to an overestimate of the time, possibly by a factor of two to three. Such an uncertainty in time is very relevant if one remembers that, for instance, a side peaked angular distribution can be transformed into forward peaked angular 
distribution if the time scale is altered by $50 \%$ or less. The conclusions is that, at present, the diffusion constants are not known better than within a factor of two and that the direct use of the Fokker-Planck Equation to the analys is of the experimental distributions may be trusted only. qual itatively.

\section{CONCLUSIONS}

The purpose of this paper has been the discussion of the time evolution along the mass asymmetry mode of the intermediate complex in heavy ion reactions. The experimental evidence of this time evolution, consisting of mass or charge distributions, angular distributions and their dependence from fragment kinetic energy has been presented. It has been proposed that this time evolution is diffusive in nature and can be described in terms of the Master Equation or the Fokker-Planck Equation. The application of both equations to the experimental data has been discussed and the results have been critically evaluated.

\section{BIBLIOGRAPHY}

"This work was supported by the U. S. Energy Research and Development Administration.

+Sloan Fellow, 1974-76.

1. A. G. Artukh, G. F. Gridnev, V. L. Mikheev, V. V. Volkov and J. Wilczynski, Nuc?. Phys. A 215, 91 (1973).

2. L. G. Moretto, D. Heunemann, R. C. Jared, R. C. Gatti and S. G. Thompson, Physics and Chemistry of Fission 1973 (Intern. Atomic Energy Agency, Vienna, 1974), Vo1. II, p. 351.

3. F. Hanappe, M. Lefort, C. Ngô, J. Peter and B. Tamain, Phys. Rev. Letters 32, 738 (1974).

4. K. L. Wolf, J. P. Unik, J. R. Huizenga, J. Birkelund, H. Freiesleben, and V. E. Viola, Phys. Rev. Letters 33, 1105 (1974).

5. B. Gatty, D. Guerreau, M. Lefort, X. Tarrago, J. Galin, B. Cauvin, J. Girard and H. Nifenecker, Nuc1. Phys. A 253, 511 (1975).

6. S. Agarwal, J. Galin, B. Gatty, D. Guerreau, M. Lefort, X. Tarrago, R. Babinet, J. Girard, and Nifenecker, private communication.

7. B. Gatty, D. Guerreau, M. Lefort, J. Pouthas, X. Tarrago, J. Galin, B. Cauvin, J. Girard and H. Nifenecker, Z. Physik A 273, 65 (1975).

8. L. G. Moretto and J. S. Sventek, Phys. Letters B 58, 26 (1975).

9. L. G. Moretto, R. P. Babinet, J. Galin and S. G. Thompson, Phys. Letters B $\underline{58}, 37$ (1975).
10. See also [7] and V. V. Volkov, Proceedings of the Intern. Conf. on Reactions between Complex Nuclei, Nashville, 1974, R. L. Robinson et al., eds. (North-Holland, Amsterdam, 1974), Vol. 2, p. 363.

11. L. G. Moretto and J. S. Sventek, Proceedings of the Symp. on Macroscopic Features of Heavy Ion Collision, Argonne, 1976 (LBL-5006).

12. W. Nörenberg, Phys. Letters B 53, 289 (1974).

13. W. Nörenberg, Z. Physik A 274, 241 (1975).

14. J. R. Nix, Nucl. Phys. 71, 1 (1965).

15. J. R. Nix, Nucl. Phys. A 130, 241 (1969).

16. D. Glas and V. Mosel, Phys. Letters B 49, 301 (1974).

17. R. A. Broglia, C. H. Dasso and A. Winther, Phys. Letters B 53, 30. (1974).

18. D. H. E. Gross and H. Kalinowski, Phys. Letters B $\underline{48}, 302$ (1974).

19. D. H. E. Gross, Nuct. Phys. A 240,472 (1975).

20. C. F. Tsang, Physica Scripta A 10, 90 (1974).

21. R. Bass, Nuc1. Phys. A 231, 45 (1974).

22. J. R, Nix and A. J. Sierk, Physics Scripta A 10 (1974).

23. J. P. Bondorf, M. I. Sobel and D. Sperber, Phys. Reports C 15, 83 (1974).

24. J. P. Bondorf, J. R. Huizenga, H. Sobel and D. Sperber, Phys. Rev. C 11, 1265 (1975).

25. C. Ngô, J. Peter, B. Tamain, M. Berlanger and F. Hanappe, preprint IPNO-RC-75-09.

26. J. S. Sventek and L. G. Moretto, Lawrence Berkeley Laboratory Report LBL-5012, Apri1 1976.

27. S. G. Thompson, L. G. Moretto, R. C. Jared, R. P. Babinet, J. Galin, M. M. Fowler, R. C. Gatti and J. B. Hunter, Physics Scripta $A$ 10 (1974).

28. L. G. Moretto, S. K. Kataria, R. C. Jared, R. Schmitt and S. G. Thompson, Nucl. Phys. A 255, 491 (1975).

29. J. Galin, L. G. Moretto, R. Babinet, R. Schmitt, R. Jared and S. G. Thompson, Nuc\}. Phys. A 255, 472 (1975).

30. R. Babinet, L. G. Moretto, J. Gal in, R. Jared, J. Moulton and S. G. Thompson, Nucl. Phys. A 258, 172 (1976).

31. L. G. Moretto, J. Galin, R. Babinet, Z. Fraenke1, R. Schmitt, R. Jared and S. G. Thompson, Nuc7. Phys. A 259, 172 (1976).

32. J. C. Jacmart, N. Frascaria, N. Poffe, P. Colombani, H. Doubre, M. Riou, J. C. Roynette and C. Stêphan, J. de Physique 36 , C5-107 (1975). 
33. J. C. Jacmart, P. Colombani, H. Doubre, N. Frascaria, N. Poffé, M. Riou, J. C. Roynett, C. Stéphan, A. Weidinger, Nucl. Phys. A 242, 175 (1975).

34. P. Colombani, N. Frascaria, J. C. Jacmart, M. Riou, C. Stéphan, H. Doubre, N. Poffé and J. C. Roynett, Phys. Letters B 55, 45 (1975).

35. J. B. Natowitz, M. N. Namboodiri and E. T. ChuTick, Phys. Rev. C 13, 171 (1976).

36. P. Braun-Muzinger, G. K. Gelbke, J. Barette, B. Zejdman, M. J. Levine, A. Gamp, H. L. Harney and T. Walcher, Phys. Rev. Letters 36, 849 (1976).

37. J. C. Roynette, H. Doubre, J. C. Jacmart, N. Poffé, these proceedings.

38. N. Frascari, J. C. Jacmart, N. Poffé, C. Stéphan, P. Colombani, H. Boubre, M. Riou and J. C. Raynette, these proceedings

39. R. Albrecht, F. J. Demond, W. Dunnweber, G. Graw, H. Ho, J. Slemmer, J. P. Wurm, these proceedings.

40. R. P. Schmitt, P. Russo, R. Babinet, R. Jared and L. G. Moretto, to be published.

41. F. Hanappe, M. Lefort, C. Ngô, J. Petêr and B. Tamain, Phys. Rev. Letters 32, 397 (1974).

42. J. Péter, C. Ngô and B. Tamain, J. de Physique Letters 36, L23 (1975).

43. J. Péter, C. Ngô and B. Tamain, Nucl. Phys. A 250, 351 (1975).

44. F. Hanappe, C. Ngô, J. Péter and B. Tamain, Proc. Intern. Conf. on Reactions between Complex Nuclei, Nashville, R. L. Robinson et al., eds. (North-Holland, Amsterdam, 1974), p. 116.

45. R. Vanderbosch, M. P. Webb and T. D. Thomas, Phys. Rev. Letters 36, 459 (1976).

46. L. G. Moretto, B. Cauvin, P. Glässel, R. Jared, P. Russo, J. Sventek and G. Wozniak, Phys. Rev. Letters 36, 1069 (1976).

47. K. L. Wolf and C. T. Roche, Proceedings of the Symp. on Macroscopic Features of Heavy Ion Collision, Argonne 1976.

48. G. Wozniak, P. Glässel, P. Russo, B. Cauvin, R. Schmitt and L. G. Moretto, to be published.

49. P. Russo, B. Cauvin, P. Glässel, R. C. Jared R. P. Schmitt, G. J. Wozniak and L. G. Moretto, Lawrence Berkeley Laboratory Report LBL -5050 .

50. Unpub7 ished work from our research group.

51. S. Ouichaoui, C. Ngô, J. Péter, F. Plasil and

B. Tamain, these proceedings.
52. M. Berlanger, F. Hanappe, C. Ngô, J. Péter, F. Plasil and B. Tamain, these proceedings.

53. Unpublished work from our research group.

54. W. V. Schroder, J. R. Birkelund, J. R. Huizenga, K. L. Wolf, J. P. Unik and V. Viola, Jr., Phys. Rev. Letters $\underline{36}, 514$ (1976).

55. J. R. Birkelund, W. V. Schröder, J. R. Huizenga, K. L. Wolf, J. P. Unik, and V. E. Viola, Jr., preprint.

56. B. Tamain, F. Plasil, C. Ngô, J. Péter, M. Berlanger and F. Hanappe, Phys. Rev. Letters 36, 18 (1976).

57. J. R. Birkelund et al., unpublished data, see a) so Ref. 47.

58. W. V. Schröder, J. R. Birkelund, J. R. Huizenga, K. L. Wolf. J. P. Unik and V. E. Viola, Jr., these proceedings.

59. K. L. Wolf, C. T. Roche and R. L. Boudrie, these proceedings.

60. J. R. Huizenga, 3. R. Birkelund, W. V. Schroder, K. C. Wolf, and V. E. Viola, Jr., these proceedings.

61. M. M. Fowler and R. C. Jared, Nucl. Instrum. Methods 124, 341 (1975). 

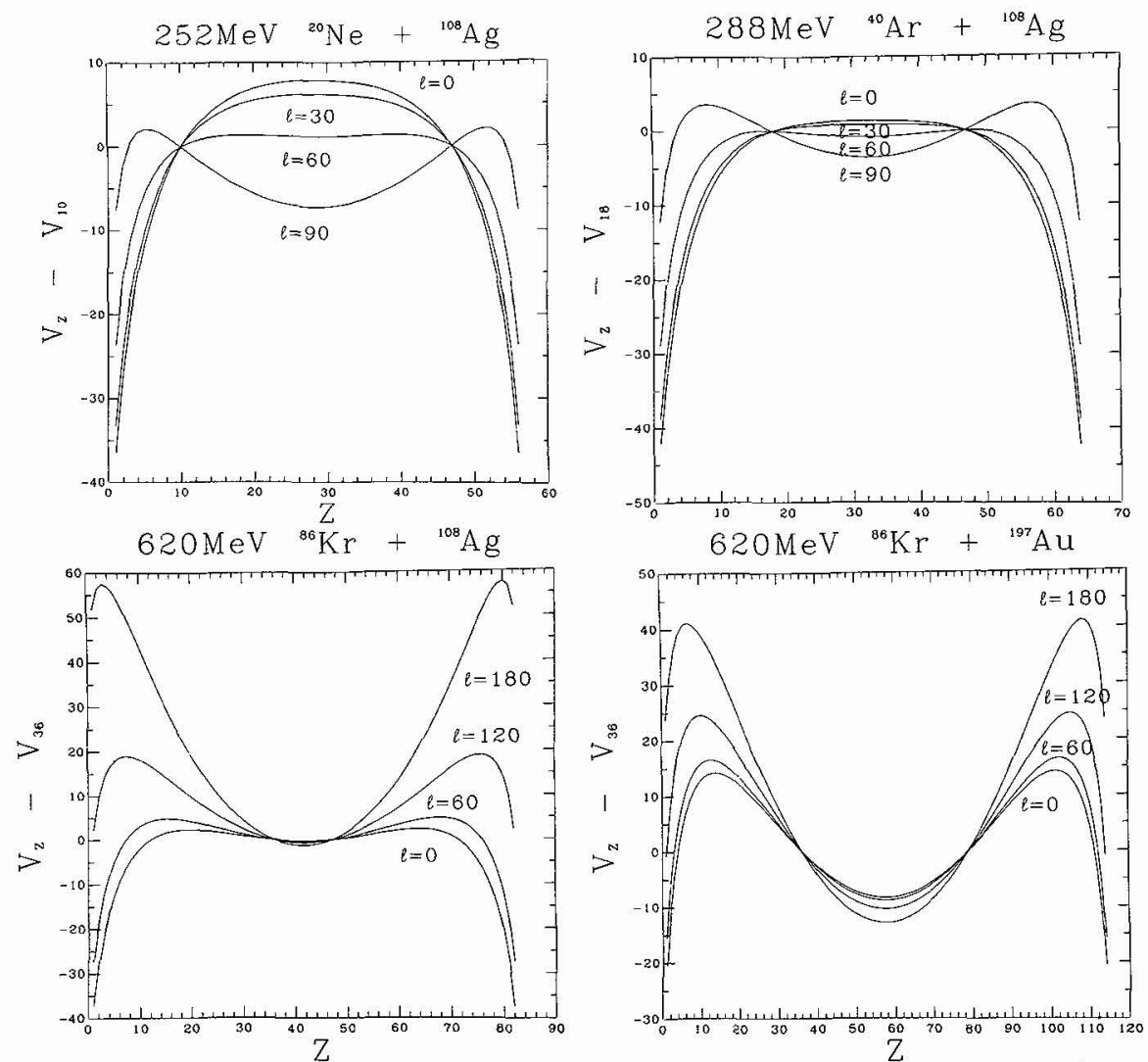

Fig. 1 (a). Ridge line potentials for the four reactions: $108 \mathrm{Ag}+{ }^{20} \mathrm{Ze},{ }^{108} \mathrm{Ag}+{ }^{40} \mathrm{Ar},{ }^{108} \mathrm{Ag}+{ }^{86} \mathrm{Kr}$ and ${ }^{197} \mathrm{Au}+86 \mathrm{Kr}$. Each curve is labeled with its own \& wave. For each curve the potential is measured relative to the injection point ( $\mathrm{Z}$ of the projectile).

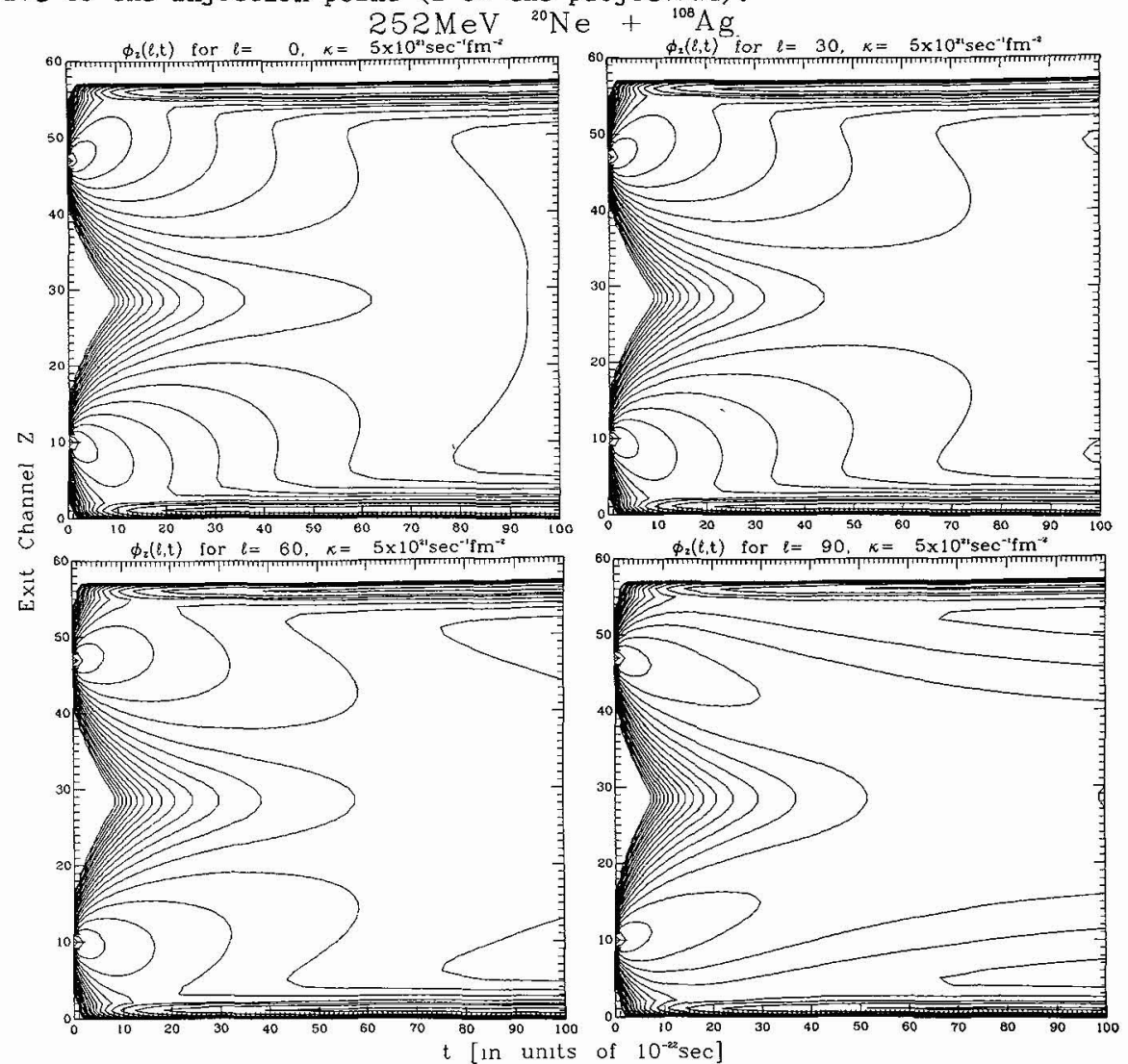

Fig, l(b). Contours of constant population in the plane defined by the mass asymetry coordinate and the time for four \& waves for the reaction $108 \mathrm{Ag}+20 \mathrm{Ne}$ at $252 \mathrm{MeV}$ bombarding energy. 


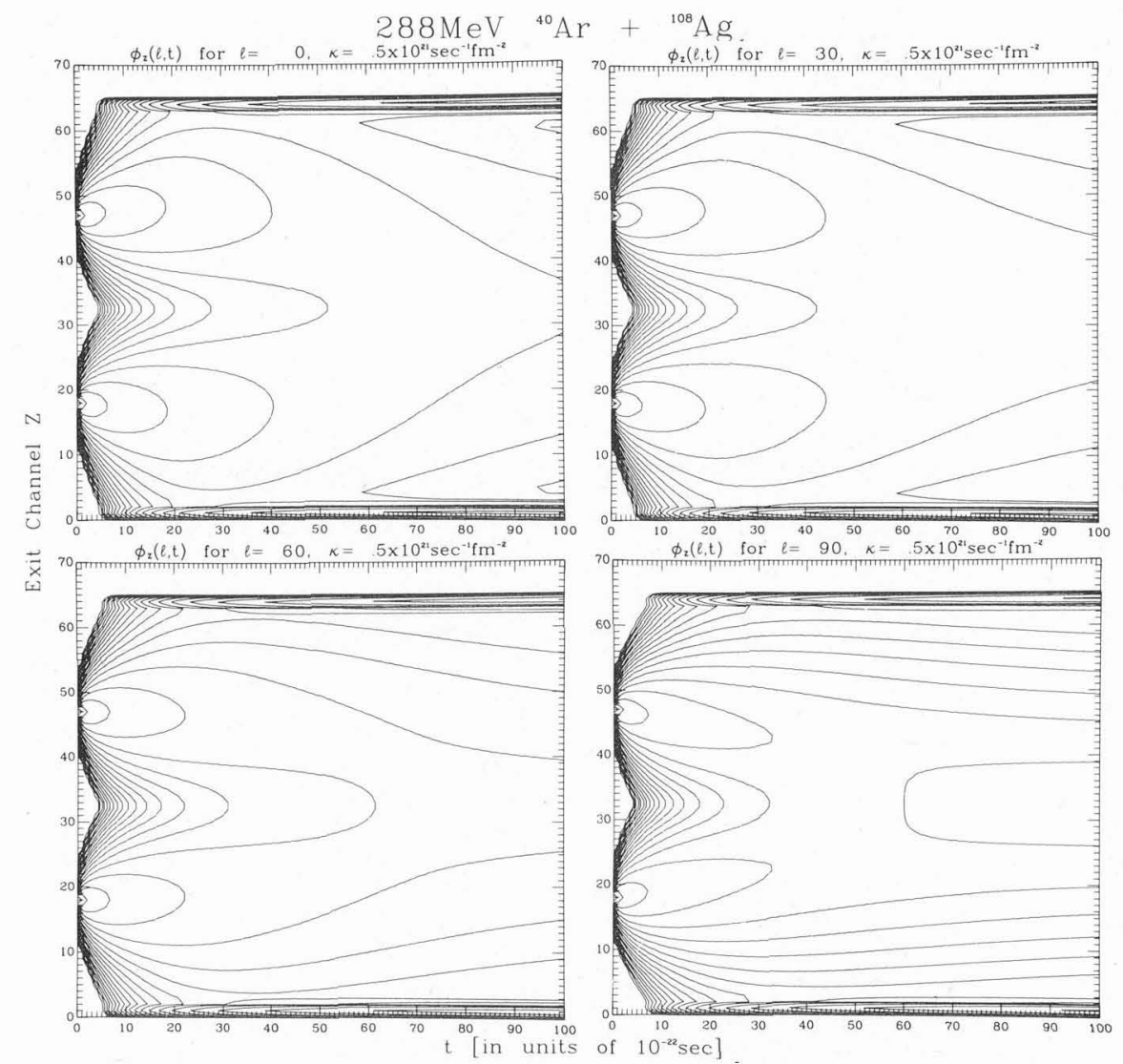

Fig. 1(c). Same as in (b) for the reaction ${ }^{108} \mathrm{Ag}+4 \mathrm{O}_{\mathrm{Ar}}$ at $288 \mathrm{MeV}$ bombarding energy.

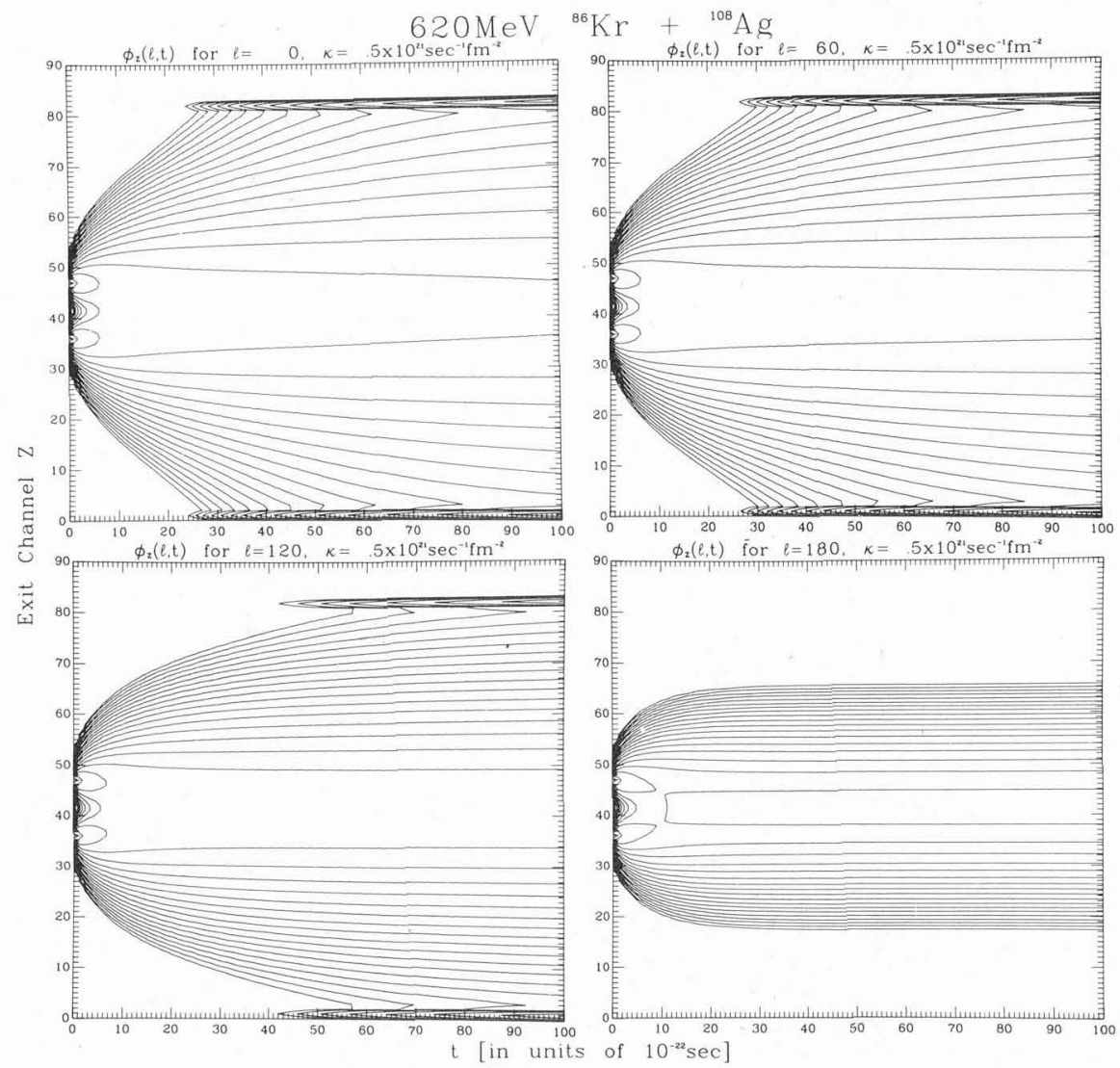

Fig. 1 (d). Same as in (b) for the reaction ${ }^{108} \mathrm{Ag}+{ }^{86} \mathrm{Kr}$ at $620 \mathrm{MeV}$ bombarding energy. 


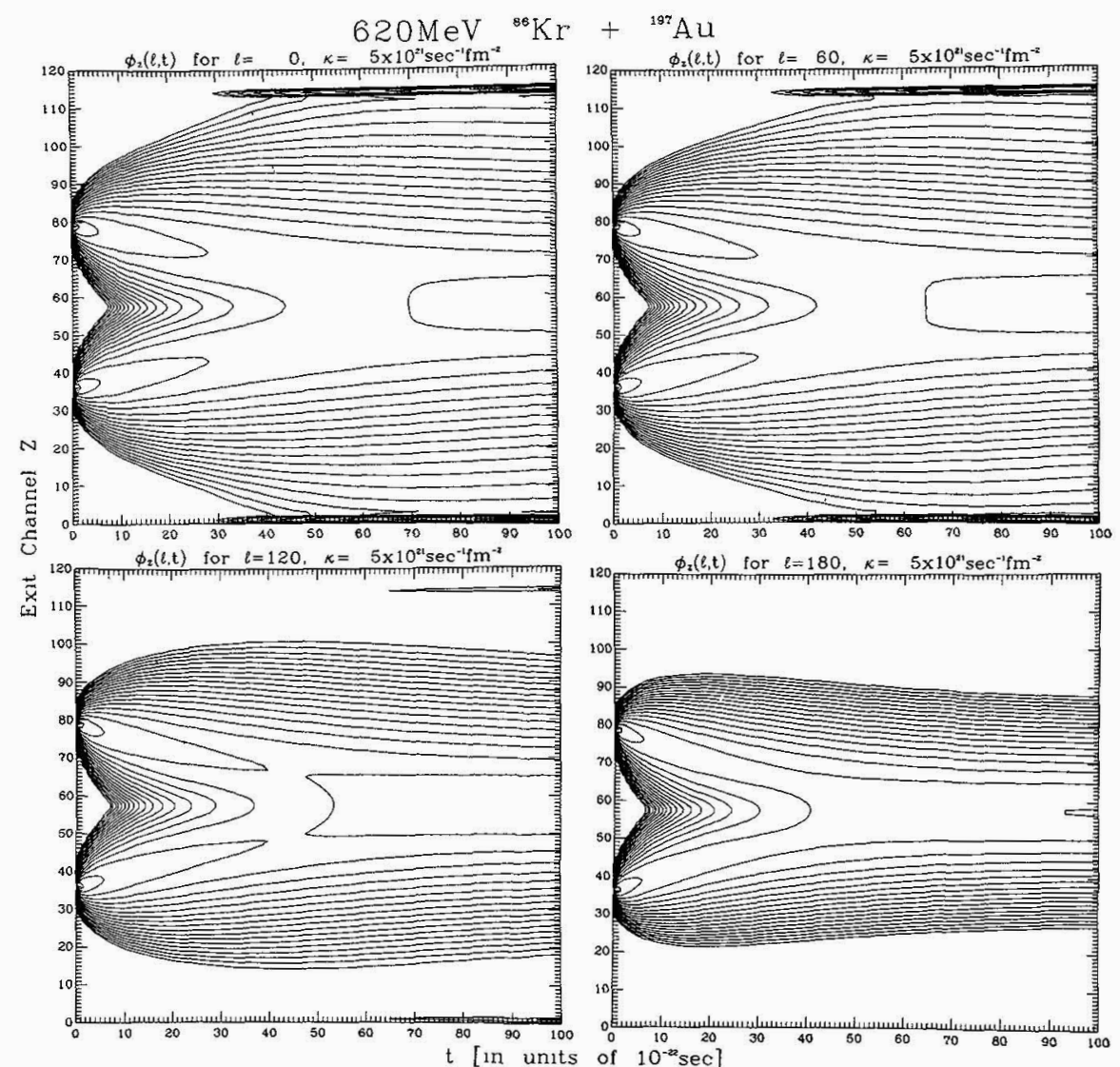

Fig. 1 (e). Same as in (b) for the reaction ${ }^{197} \mathrm{Au}+{ }^{86} \mathrm{Kr}$ at $620 \mathrm{MeV}$ bombarding energy. The sharpening of the population distributions at high $\ell$ values is due to a drastic reduction in temperature because of the energy tied up in rotational energy.
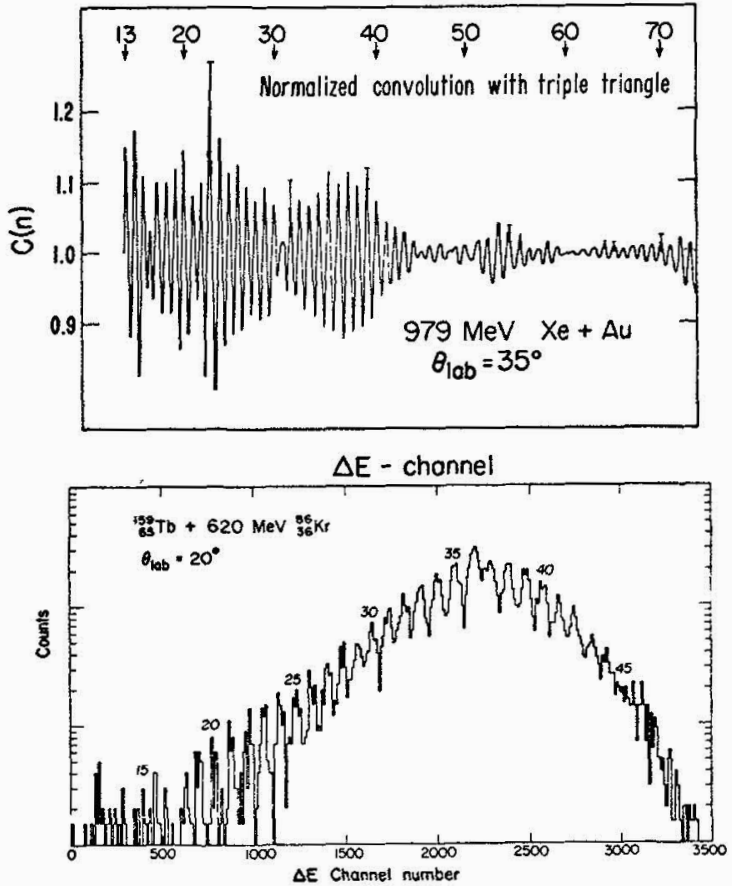

Fig. 2(a). Cut of the $\Delta E-E$ map at a fixed $E$ value for the reaction $159 \mathrm{~Tb}+86 \mathrm{Kr}$ at $620 \mathrm{MeV}$ bombarding energy [46]. Individual a tomic numbers are visua1ly resolved up to $Z=45$. (b) Convolution of a constant $E$ cut and $\Delta E-E$ map with three triangular peaks as a function of the phase for the reaction $197_{\mathrm{Au}+}$ $136 \mathrm{Xe}$ at $980 \mathrm{MeV}$. Whenever the three peaks match the positions of three consecutive peaks in the cut, a maximum appears in the convolution. In this case atomic members are identified up to and above $Z=60$.

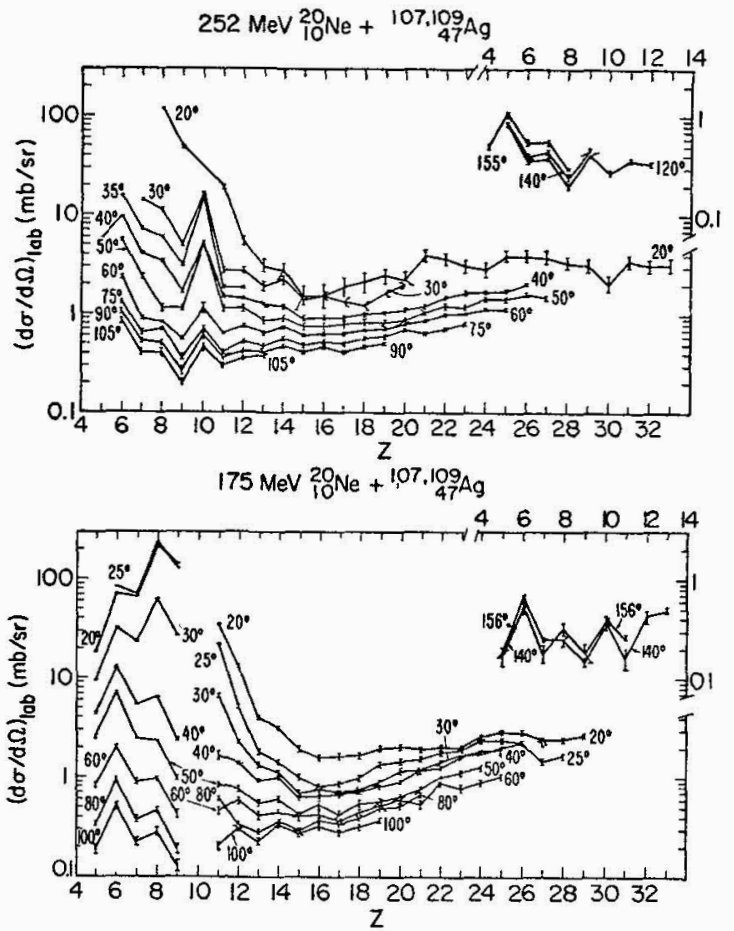

Fig. 3. Laboratory cross sections as a function of $\mathrm{Z}$ for various laboratory angles for the reaction $107,{ }^{109} \mathrm{Ag}+20 \mathrm{Ne}$ at 175 and $252 \mathrm{MeV}$ bombarding energies $[30]$. 

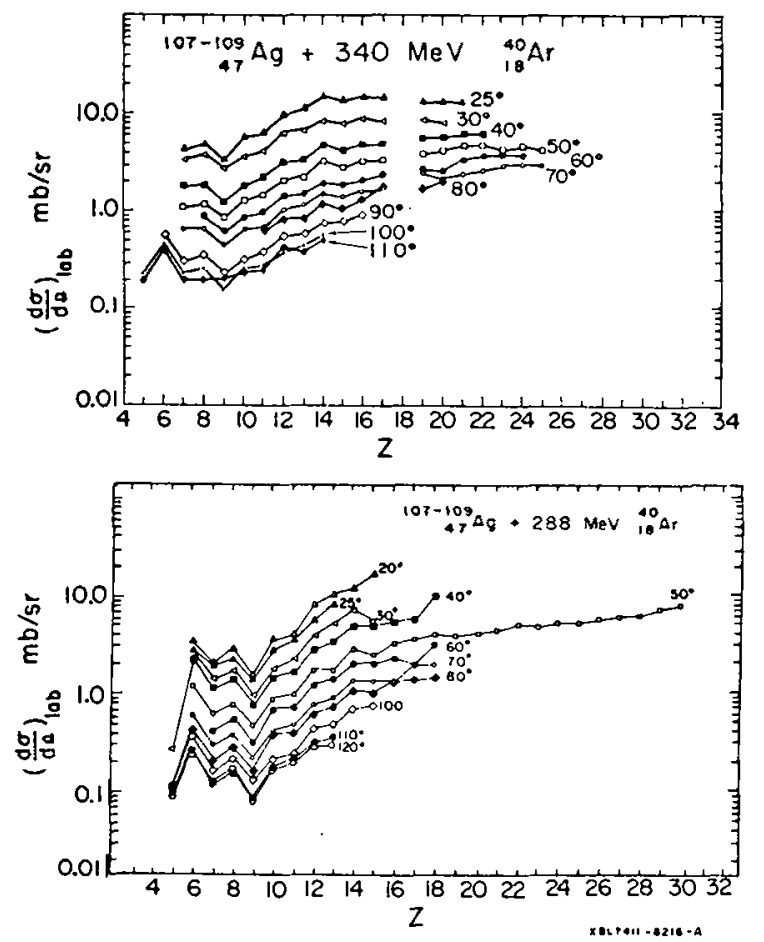

Fig. 4. Same as in Fig. 3 for the reaction $10 \%, 109 \mathrm{Ag}+40 \mathrm{Ar}$ at 288 and $340 \mathrm{MeV}$ bombarding energies [29].

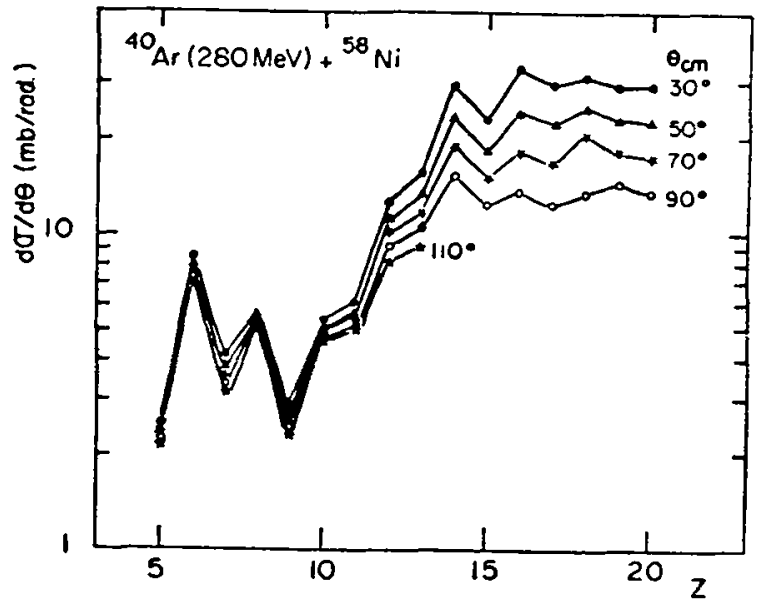

Fig. 5. Same as in Fig. 3 for the reaction $58 \mathrm{Ni}+40_{\mathrm{Ar}}$ at $280 \mathrm{MeV}$ bombarding energy [5].
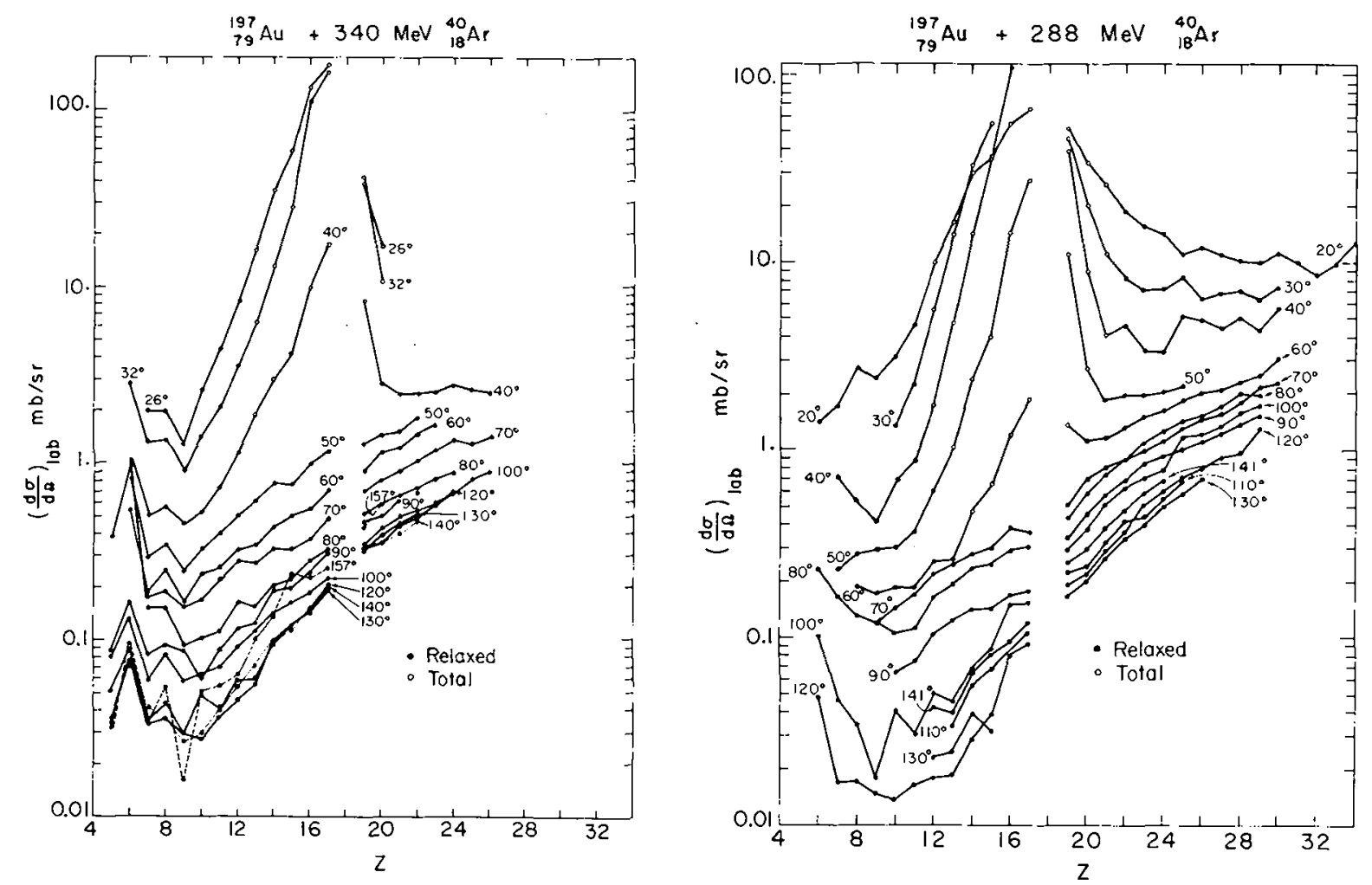

Fig. 6. Same as in Fig. 3 for the reaction ${ }^{197} \mathrm{Au}+40 \mathrm{Ar}$ at 288 and $340 \mathrm{MeV}$ hombarding energies [31]. 

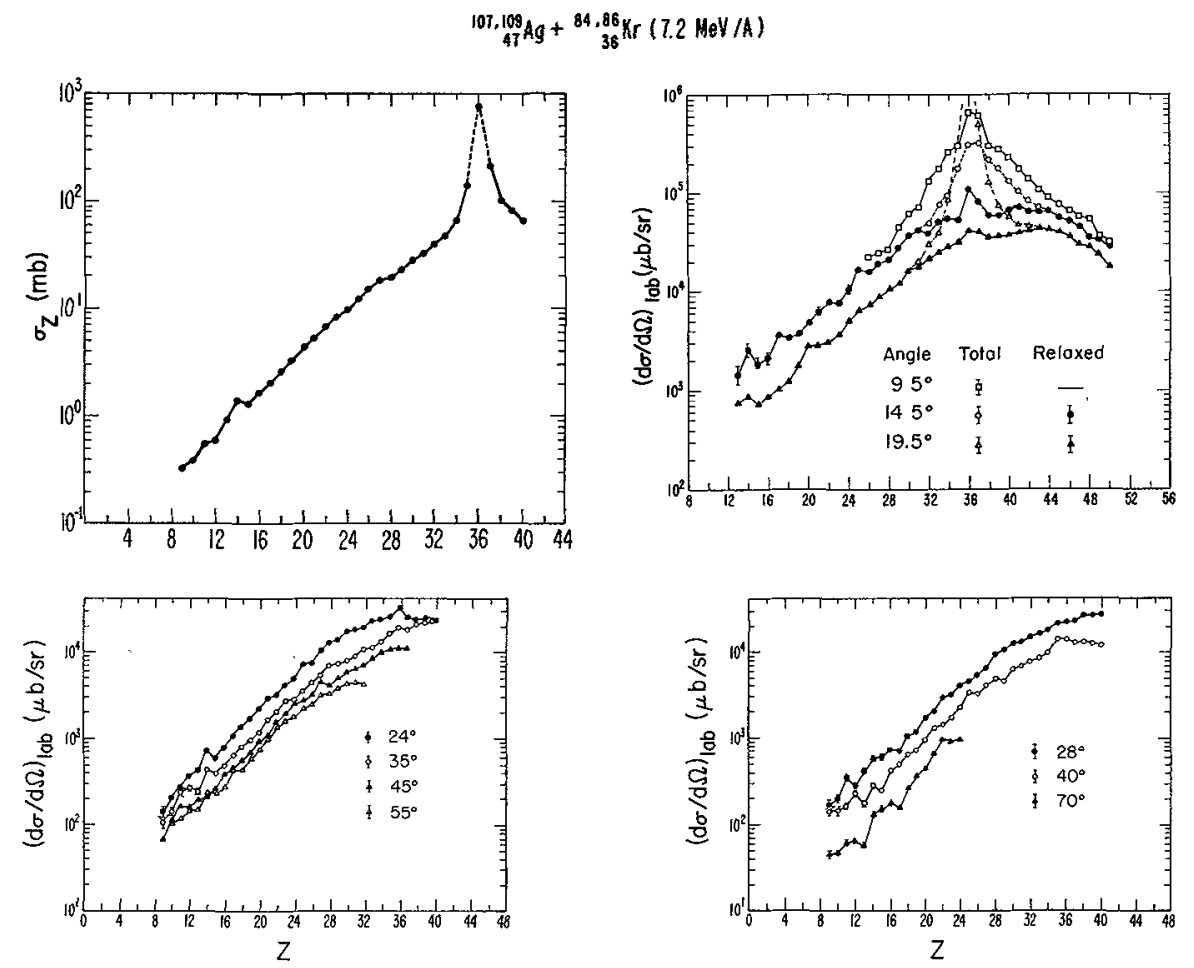

Fig. 7. (b), (c), (d). Same as in Fig. 3 for the reaction ${ }^{107,109} \mathrm{Ag}+{ }^{86} \mathrm{Kr}$ at $620 \mathrm{MeV}$ bombarding energy [40]. (a) Total cross section integrated over angle from $\theta_{\mathrm{Lab}}=10^{\circ}$ to $\theta_{\mathrm{Lab}}=55^{\circ}$ as a function of $\mathrm{z}$.

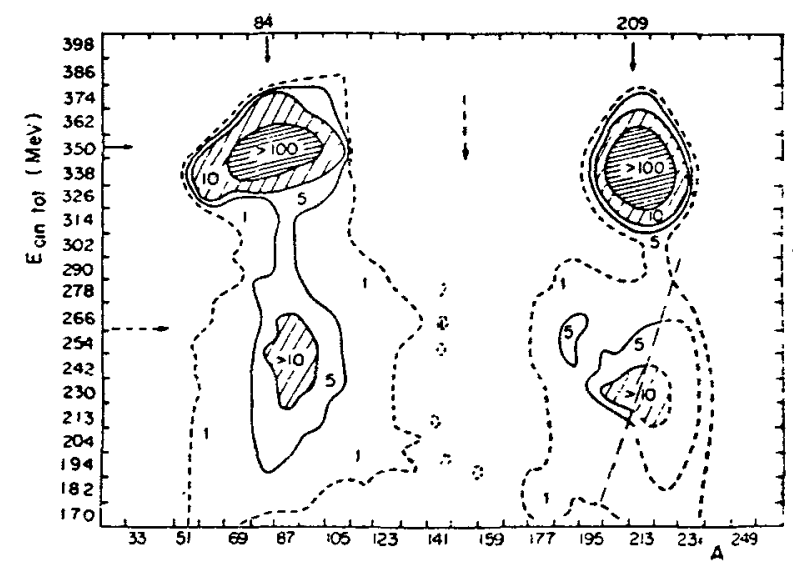

Fig. 8. Contour map for the cross section in the plane defined by the total fragment kinetic energy and by the fragment mass, for the reaction ${ }^{209} \mathrm{Bi}+{ }^{86} \mathrm{Kr}$ at $500 \mathrm{MeV}$ bombarding energy [3].

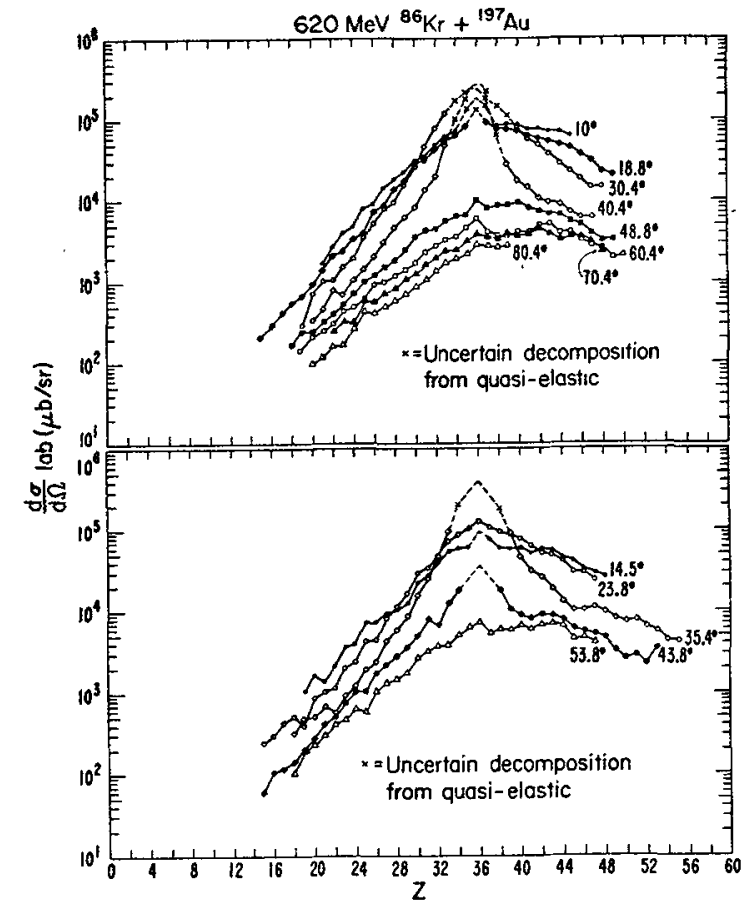

Fig. 9 . Same as in Fig. 3 for the reaction
${ }^{9} \mathrm{Au}+86 \mathrm{Kr}$ at $620 \mathrm{MeV}$ bombarding energy $[46]$ 


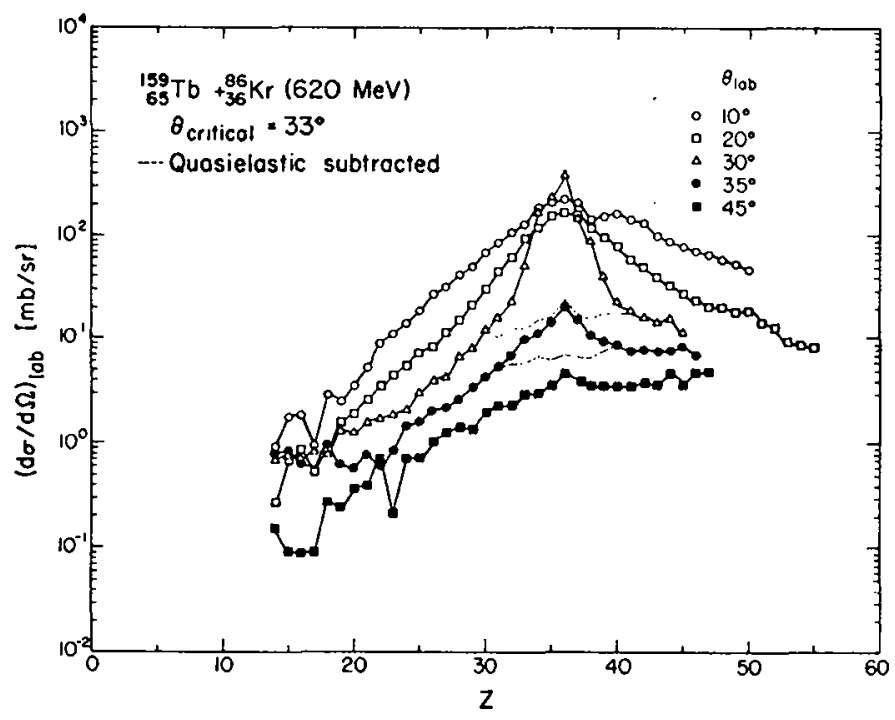

Fig. 10. Same as in Fig. 3 for the reaction ${ }^{159} \mathrm{~Tb}+{ }^{86} \mathrm{Kr}$ at $620 \mathrm{MeV}$ hombarding energy [48].
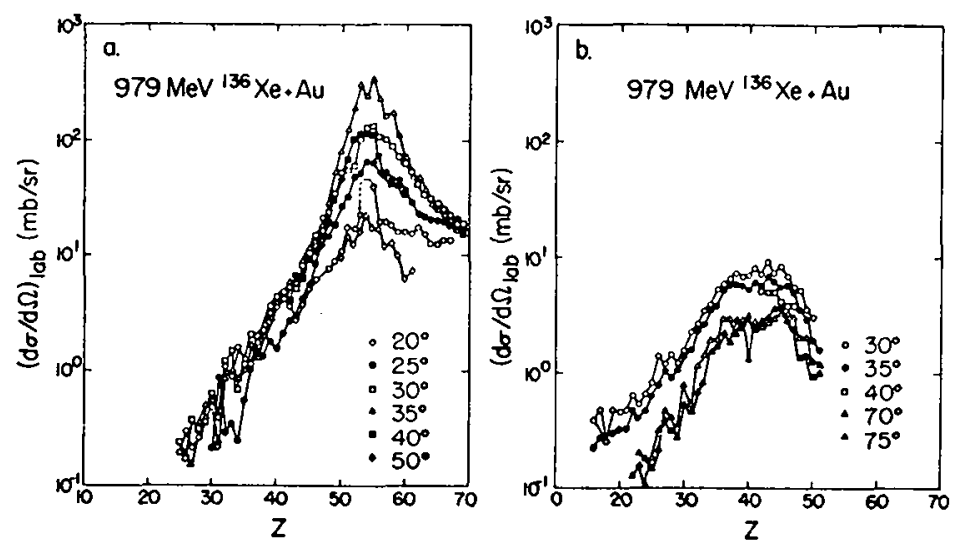

Fig. 11. (a) Same as in Fig. 3 for the reaction ${ }^{197} \mathrm{Au}+{ }^{136}$ Xe at $980 \mathrm{MeV}$ bombarding energy [49]. (b) $z$ distributions for the secondary fission of the quasi Au fragment.

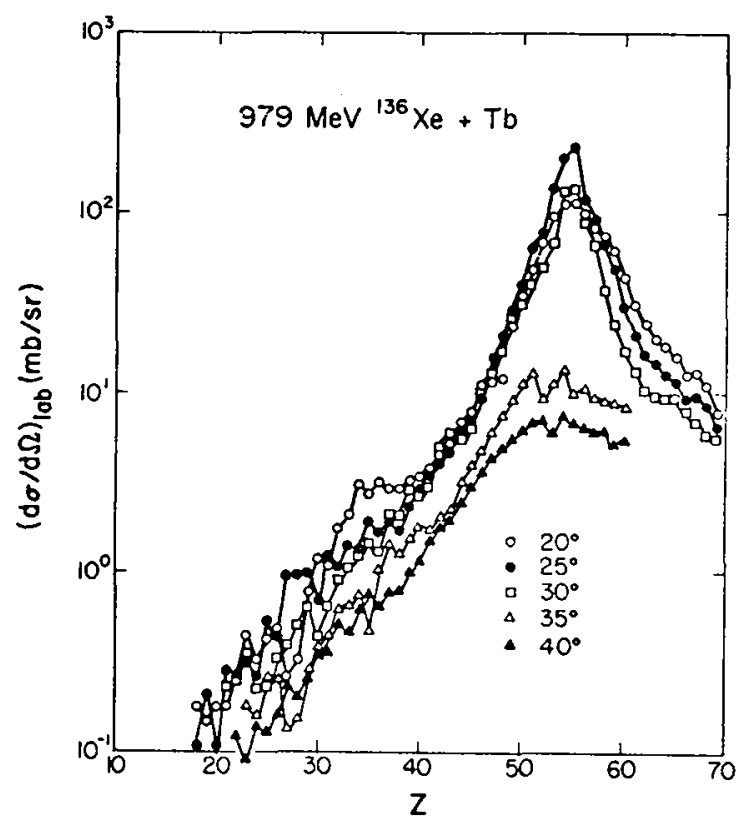

Fig. 12. Same as in Fig. 3 for the reaction ${ }^{159} \mathrm{~Tb}+{ }^{136} \mathrm{Xe}$ at $980 \mathrm{MeV}$ bombarding energy [29]. 


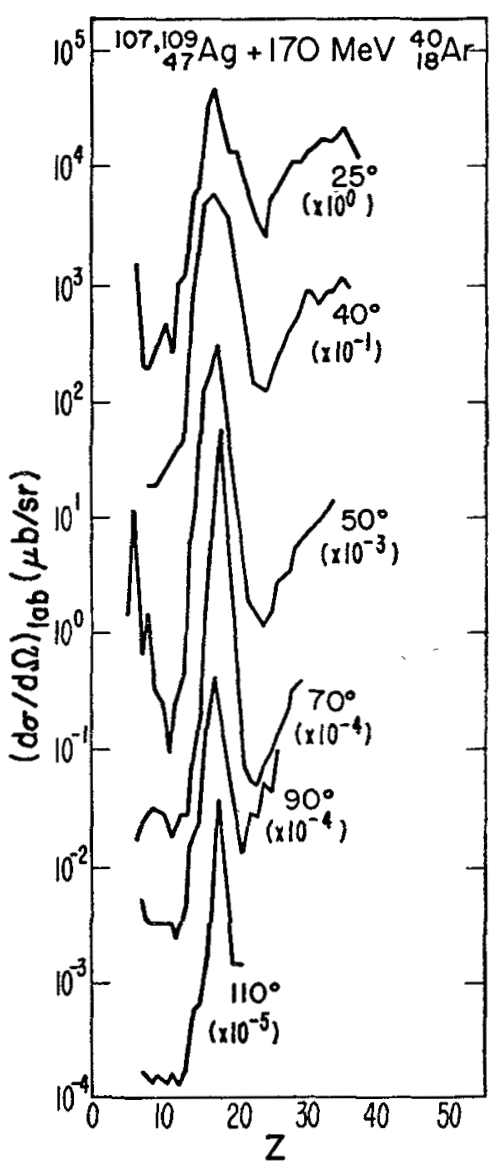

Fig. 13. Same as in Fig. 3, for the reaction $107,109 \mathrm{Ag}+40 \mathrm{Ar}$ at $170 \mathrm{MeV}$ bombarding energy $[29]$.

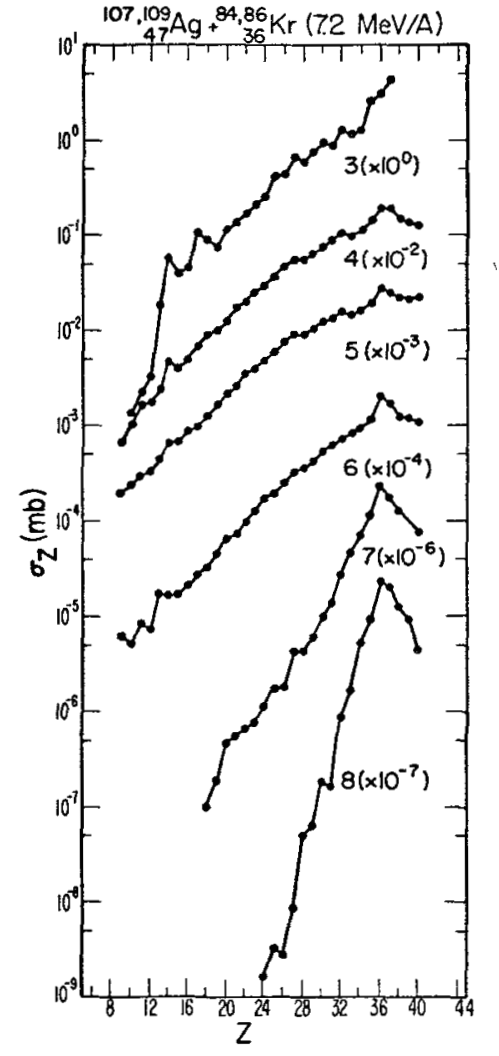

Fig. 14. Z distributions (integrated over angle from $\theta_{1 a b}=10^{\circ}$ to $\theta_{1 a b}=55^{\circ}$ ) for various kinetic energy bins for the reaction $107,{ }^{109} \mathrm{Ag}+86_{\mathrm{Kr}}$ at $620 \mathrm{MeV}$ bombarding energy [40]. The bins are $20 \mathrm{MeV}$ wide. The bin number multiplied by 20 gives the upper energy of the bin in $\mathrm{MeV}$ at $\mathrm{z}=36$. See text for more detailed explanation. Notice the narrow quasi-elastic $Z$ distribution at the higher kinetic energy bins and the broader $\mathrm{Z}$ distributions at lower energies.

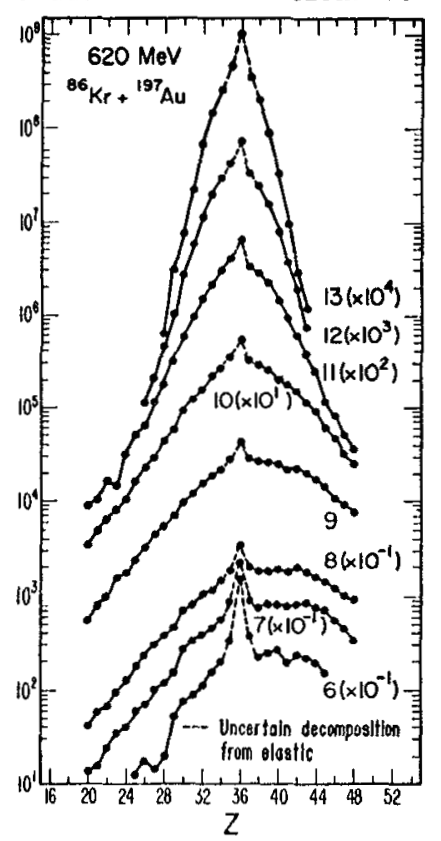

Fig. 15. (a) Total cross section, integrated over angle from $\theta_{1 \mathrm{ab}}=10^{\circ}$ to $\theta$ iab $=80^{\circ}$ as a function of $\mathrm{z}$ for the reaction $197 \mathrm{Au}+86_{\mathrm{Kr}}$ at $620 \mathrm{MeV}$ bombarding energy $[46]^{\mathrm{ab}}$ (b) $Z$ distributions, integrated over angle for various kinetic energy bins. The bin number multiplied by 20 gives the upper energy for the bin in $\mathrm{MeV}$ at $\mathrm{Z}=36$. Notice the smooth increase in width with decreasing bin energy. 


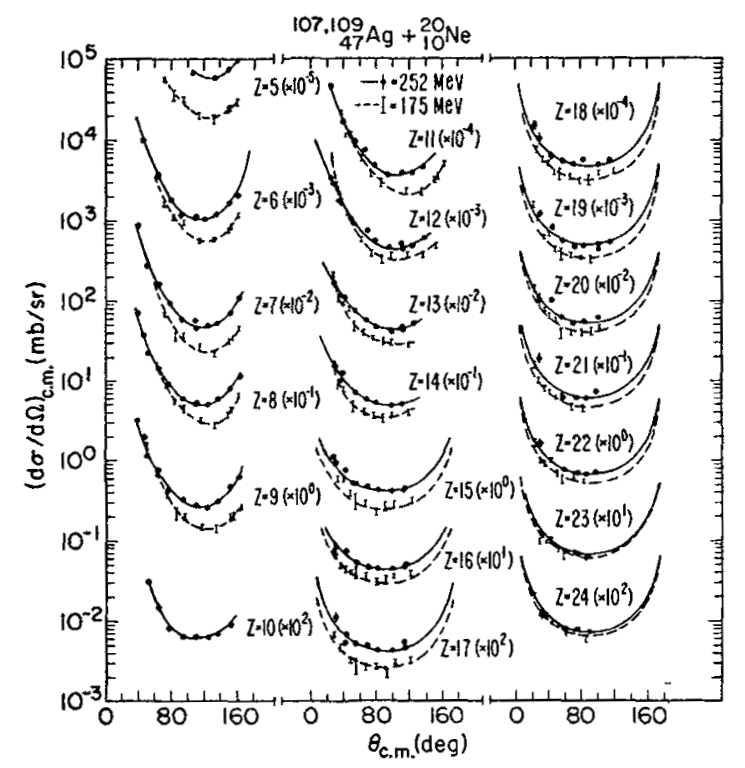

Fig. 16. Center of mass angular distributions as a functiog of fragment atomic number for the reaction $187,109 \mathrm{Ag}+2 \mathrm{O}_{\mathrm{Ne}}$ at 175 and $252 \mathrm{MeV}$ bombarding energies [30].

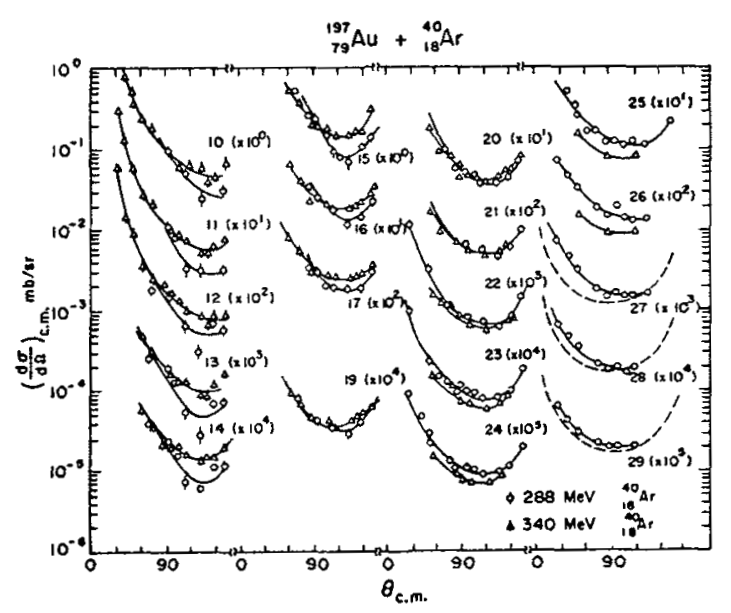

Fig. 17. Same as in Fig. 16 for the reaction $199 \mathrm{Au}+40 \mathrm{Ar}$ at 288 and $340 \mathrm{MeV}$ bombarding energies [31].

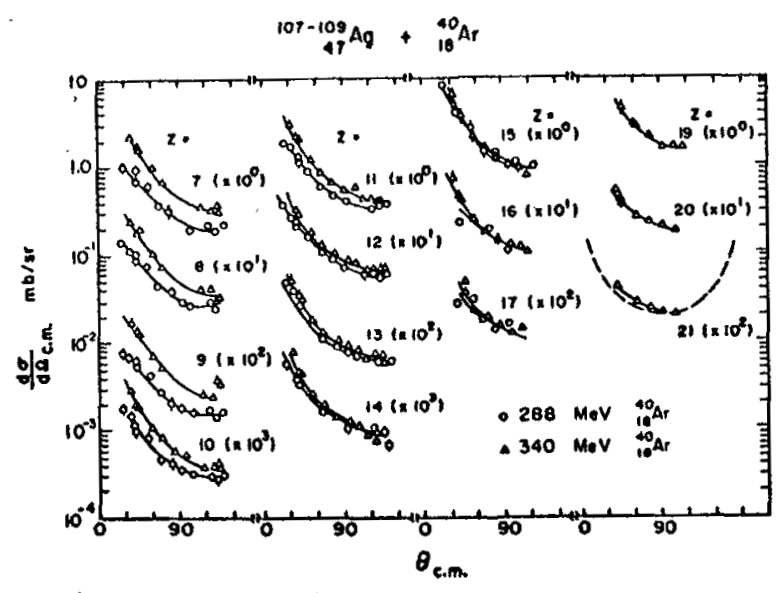

Fig. 18. Same as in Fig. 16 for the reaction $107,109 \mathrm{Ag}+40 \mathrm{Ar}$ at 288 and $340 \mathrm{MeV}$ bombarding energies [29].

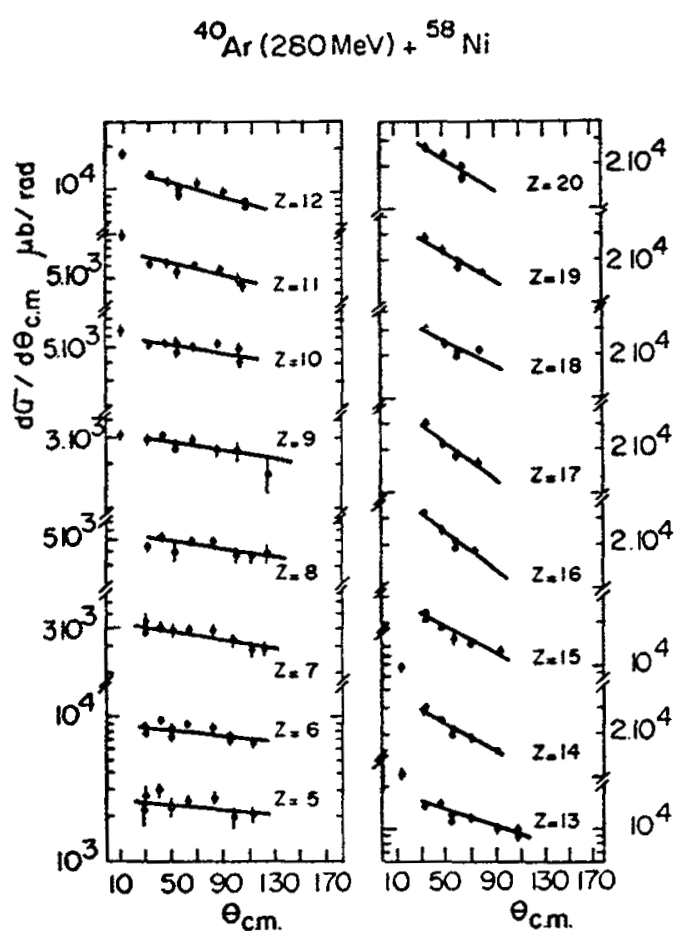

Fig. 19. Same as in Fig. 16 for the reaction $58_{\mathrm{Ni}}+40_{\mathrm{Ar}}$ at $280 \mathrm{MeV}$ bombarding energy $|5|$. Notice that, contrary to the previous figures the quantity do/d $\theta$ is plotted rather than $\frac{\mathrm{d} \sigma}{\mathrm{d} \Omega}$.
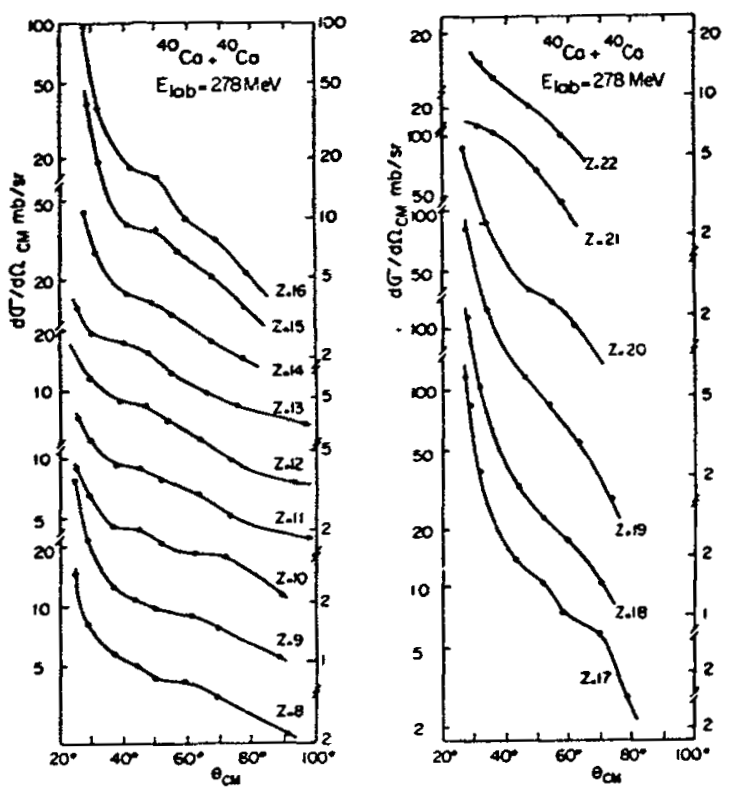

Fig. 20. Same as in Fig. 16 for the reaction $4 \mathrm{Cq}^{\mathrm{g}}+4 \mathrm{H}_{\mathrm{Ca}}$ at $278 \mathrm{MeV}$ bombarding energy. 


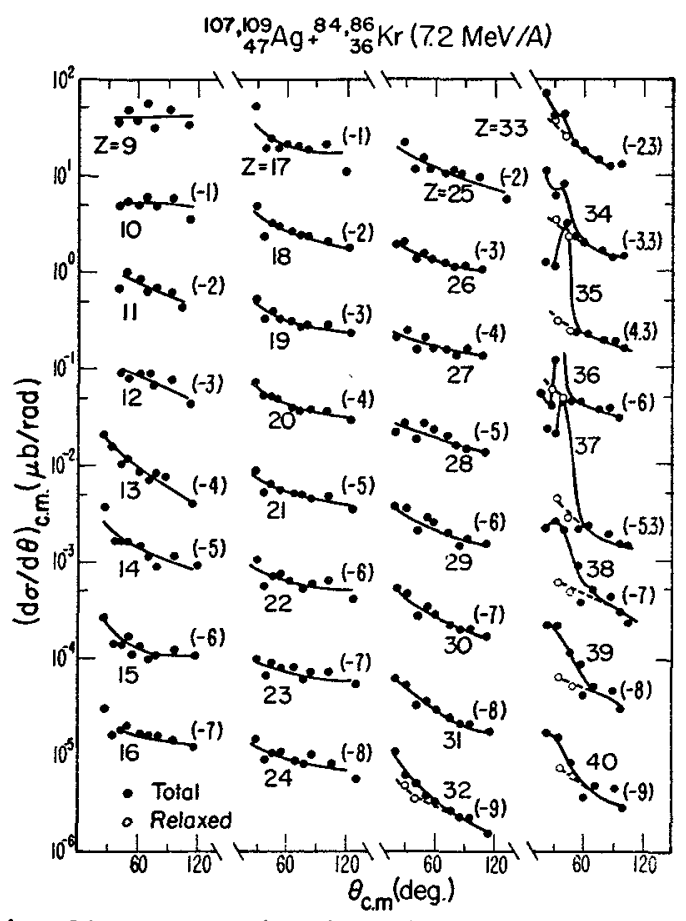

Fig. 21. Same as in Fig. 16 for the reaction $107,109 \mathrm{Ag}+86 \mathrm{Kr}$ at $620 \mathrm{MeV}$ bombarding energy [40]. The quantity $\frac{\mathrm{d} \sigma}{\mathrm{d} \theta}$ is plotted.
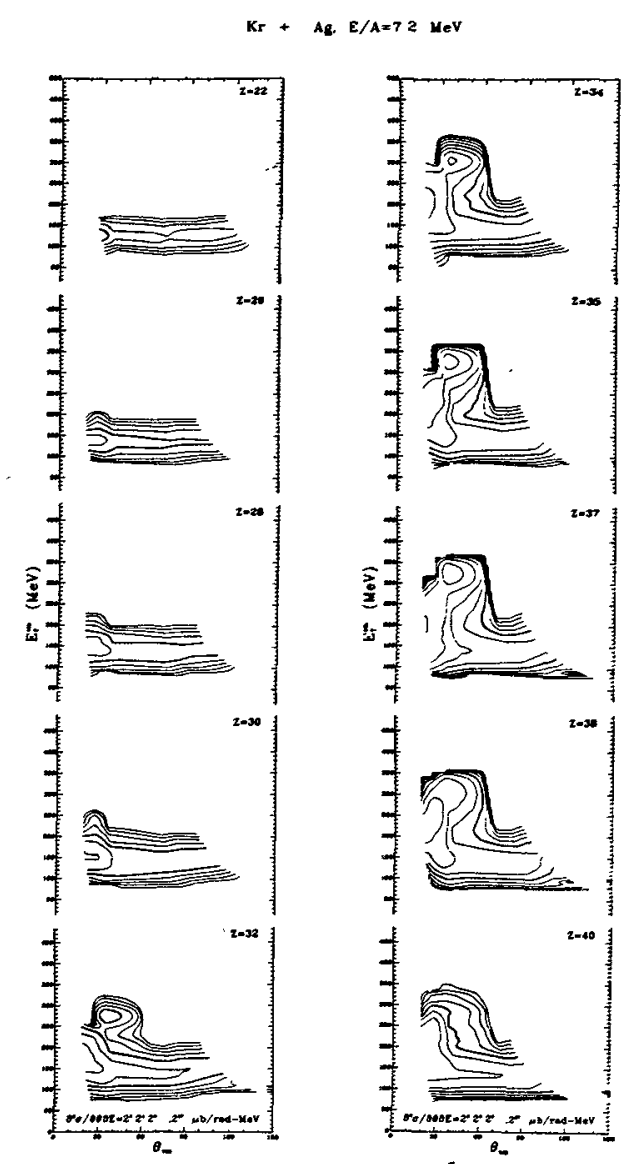

Fig.22. Contours of constant $\frac{d \sigma}{d \theta}$ in the plane defined by the fragment total kinetic energy (center of mass) and by the center of mass angle (Wilczynski plot) for a selected number of fragments for the reaction $107,109 \mathrm{Ag}+86_{\mathrm{Kr}}$ at $620 \mathrm{MeV}$ bombarding energy [40].

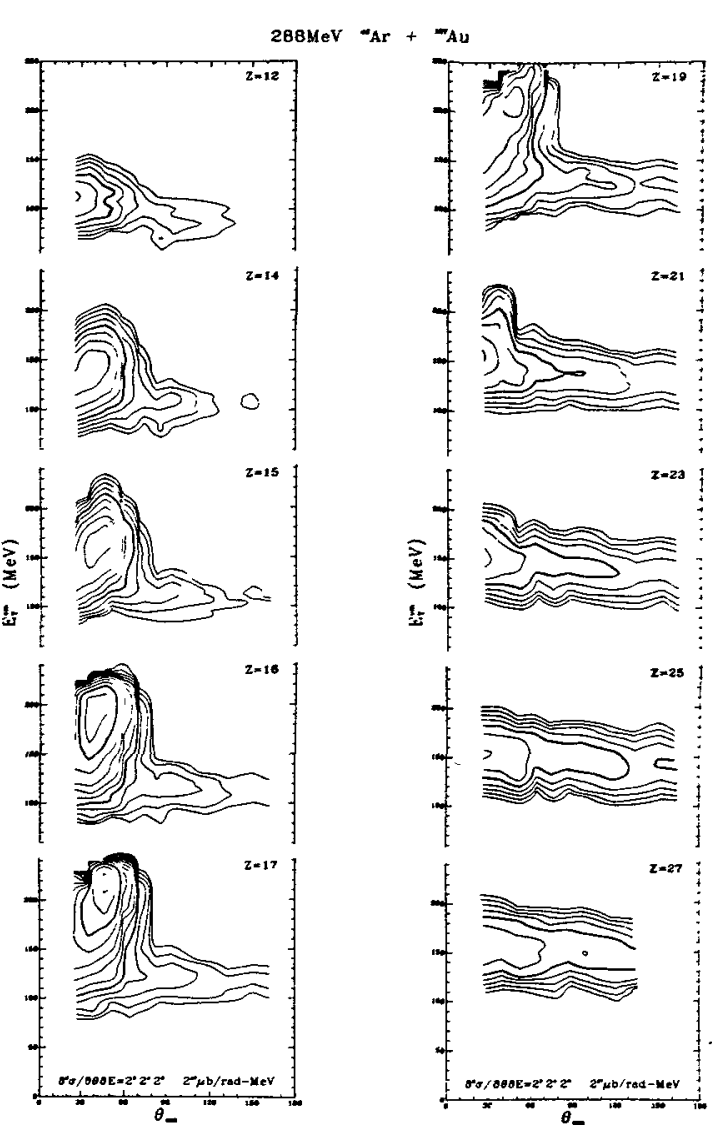

Fig.23. Same as in Fig. 22 for the reaction $197 \mathrm{Au}+40_{\mathrm{Ar}}[31]$ at $288 \mathrm{MeV}$ bombarding energix .

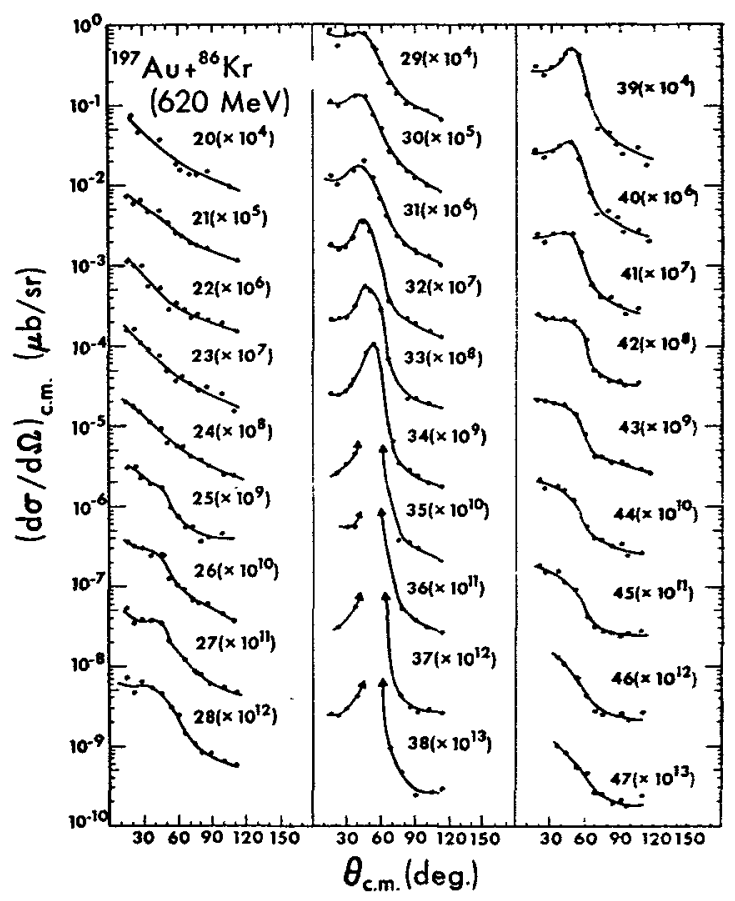

Fig. 24. Same as in Fig. 16 for the reaction $199 \mathrm{Au}+86 \mathrm{Kr}$ at $620 \mathrm{MeV}$ bombarding energy [4.6]. 


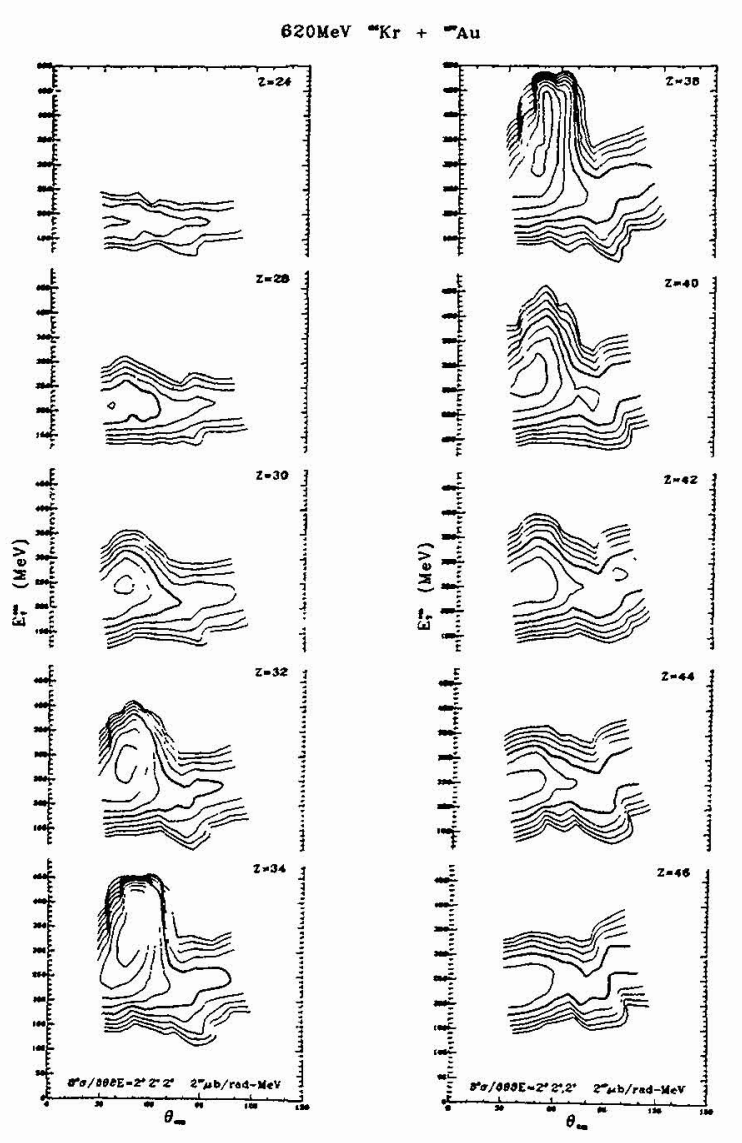

Fig. 25. Same as in Fig.22 for the reaction $197 \mathrm{Au}+86 \mathrm{Kr}$ at $620 \mathrm{MeV}$ bombarding energy [46].

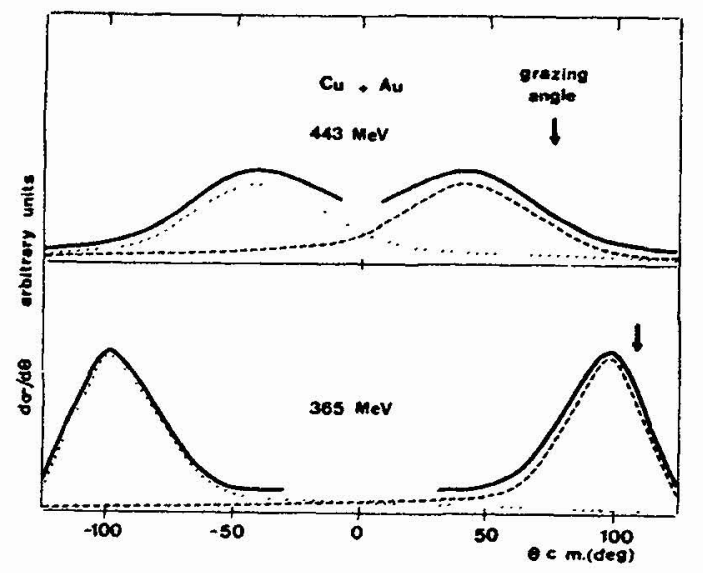

Fig. 26. Decomposition of the angular distribution $\mathrm{d} \sigma / \mathrm{d} \theta$ for the reaction $197 \mathrm{Au}+63 \mathrm{Cu}$ ar 365 and $443 \mathrm{MeV}$ bombarding energies showing the onset of orbiting.

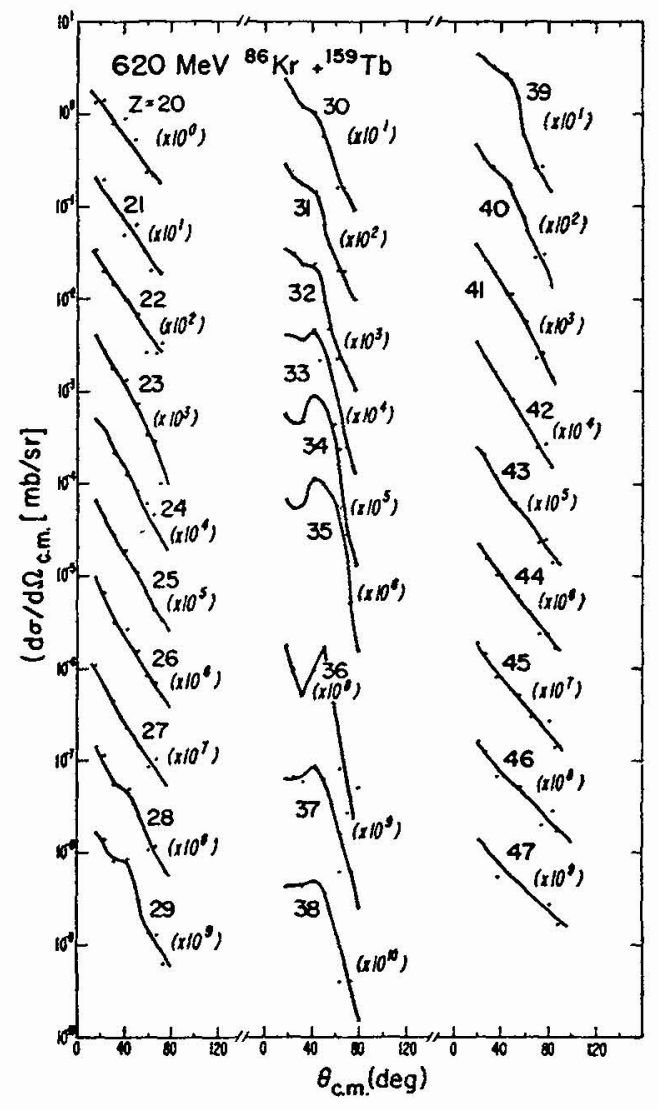

Fig.27. Same as in Fig.16 for the reaction $159 \mathrm{~Tb}+86 \mathrm{Kr}$ at $620 \mathrm{MeV}$ bombarding energy [48].

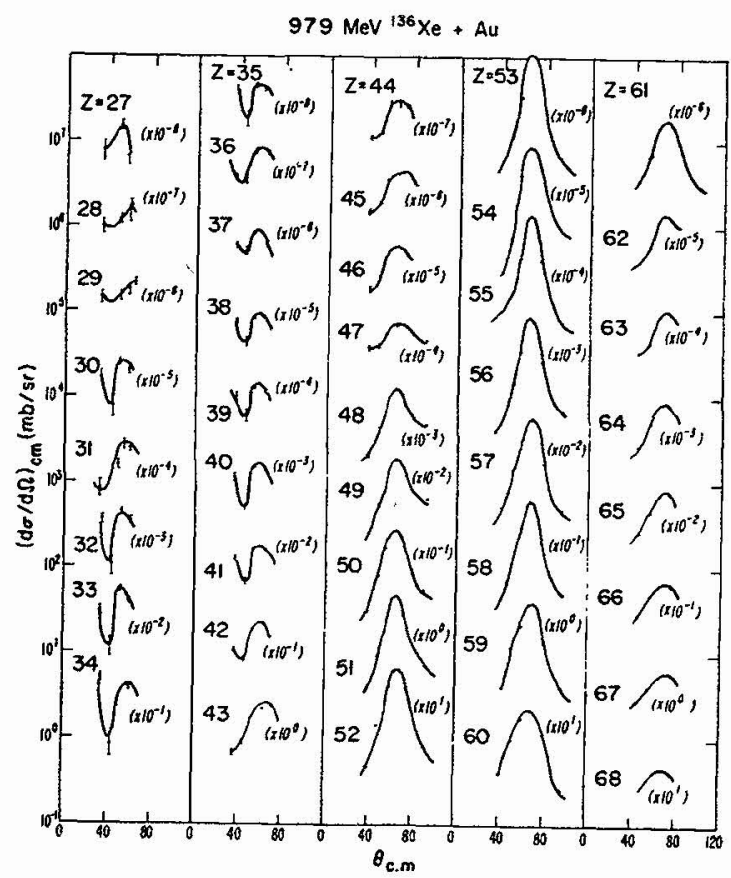

Fig. 28. Same as in Fig. 16 for the reaction $197 \mathrm{Au}+{ }^{36}$ at $980 \mathrm{MeV}$ bombarding energy [49]. 

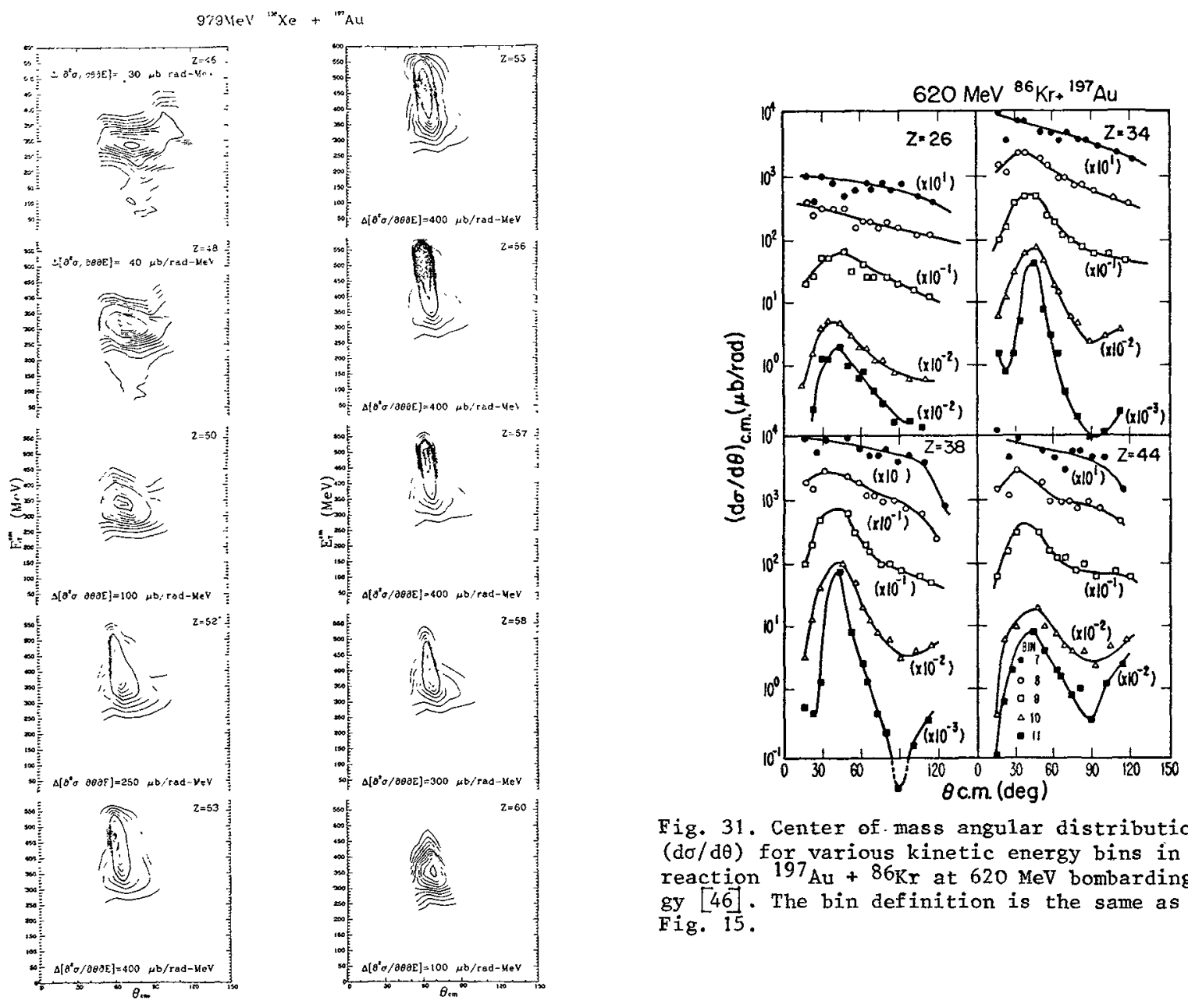

Fig. 31. Center of mass angular distributions $(\mathrm{d} \sigma / \mathrm{d} \theta)$ for various kinetic energy bins in the reaction $197 \mathrm{Au}+86 \mathrm{Kr}$ at $620 \mathrm{MeV}$ bombarding energy [46]. The bin definition is the same as in Fig. 15 .

Fig. 29. Same as in Fig. 22 for the reaction $197 \mathrm{Au}+136 \mathrm{Xe}$ at $980 \mathrm{MeV}$ bombarding energy [49].
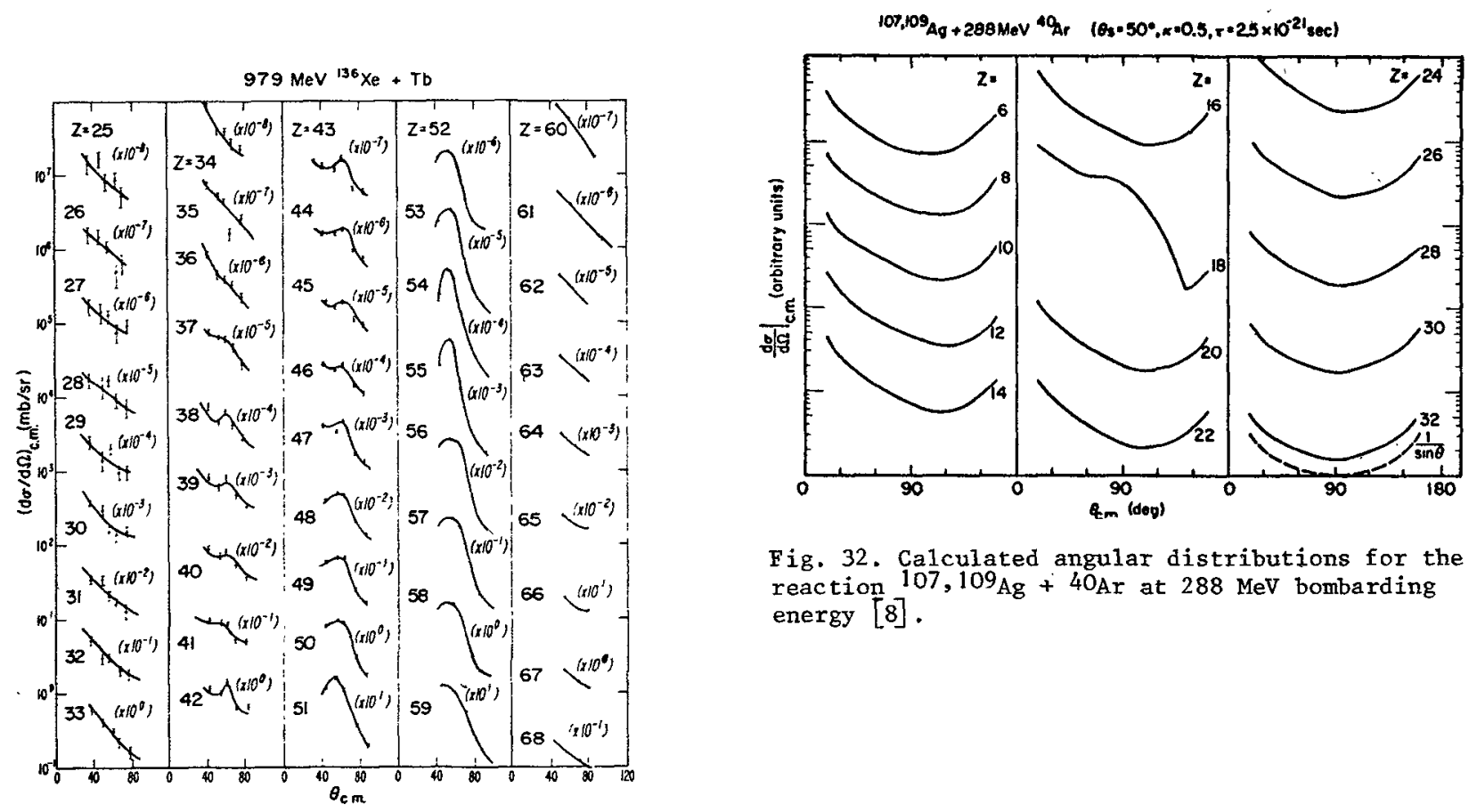

Fig. 32. Calculated angular distributions for the reaction $107,109 \mathrm{Ag}+40 \mathrm{Ar}$ at $288 \mathrm{MeV}$ bombarding energy [8].

Fig. 30. Same as in Fig. 16 for the reaction. $159 \mathrm{~Tb}+136 \mathrm{Xe}$ at $980 \mathrm{MeV}$ bombarding energy [50]. 


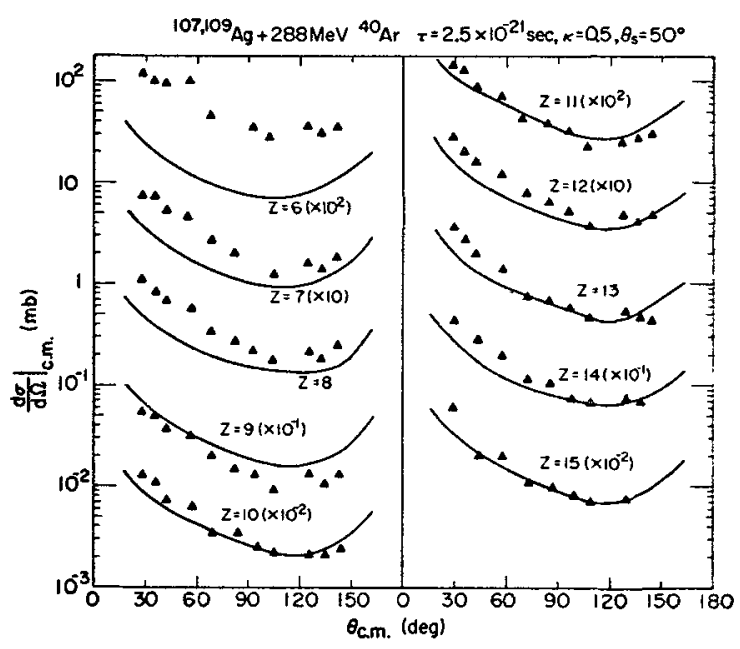

Fig. 33. Absolute comparison between calculated and experimental angular distributions for the reaction $107,109 \mathrm{Ag}+{ }^{40} \mathrm{Ar}$ at $288 \mathrm{MeV}$ bombarding energy [8].
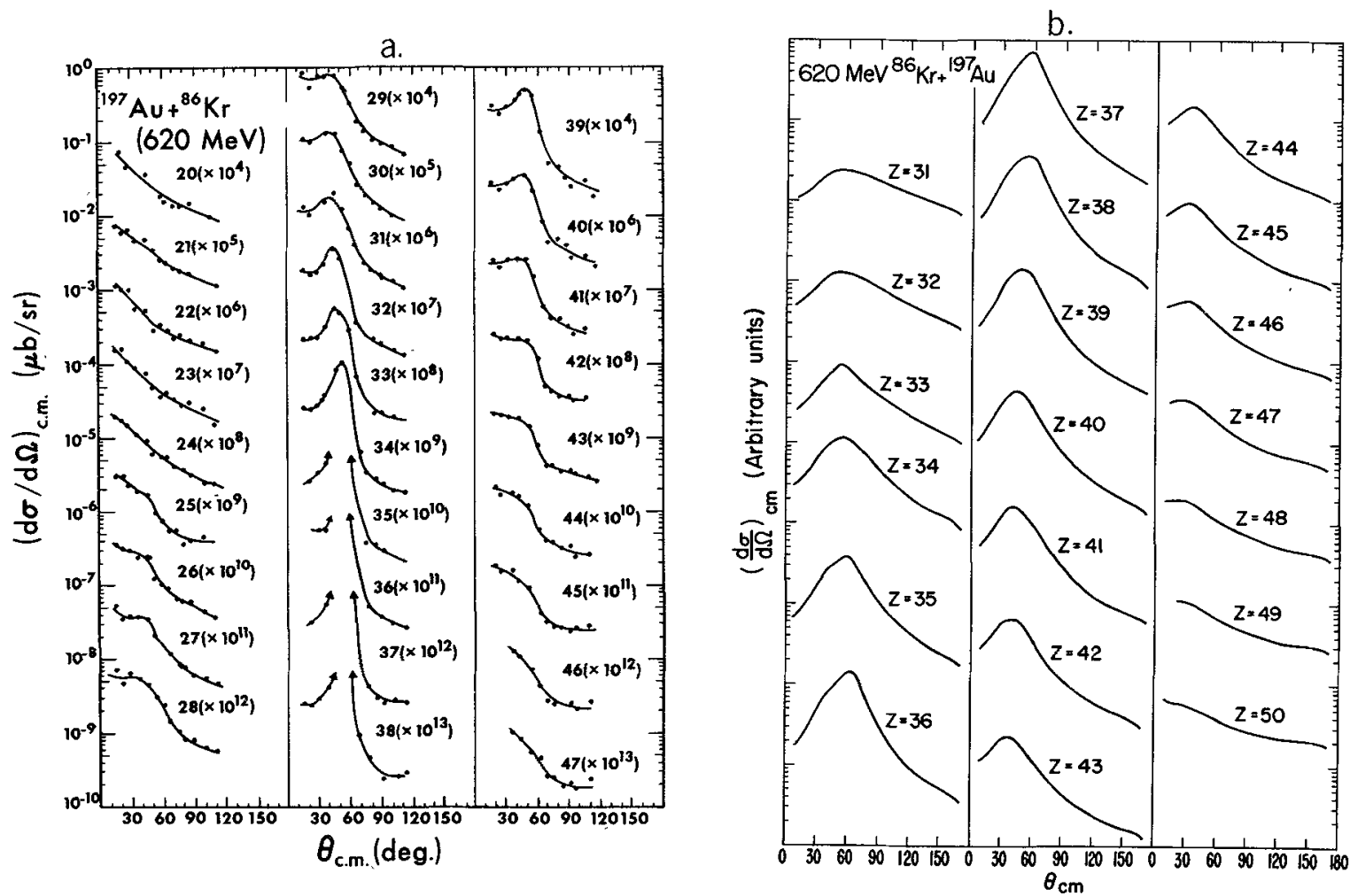

Fig. 34. (a), (b) Comparison between experimental and calculated angular distributions for the reaction ${ }^{197} \mathrm{Au}+86 \mathrm{Kr}$ at $620 \mathrm{MeV}$ bombarding energy [26]. 

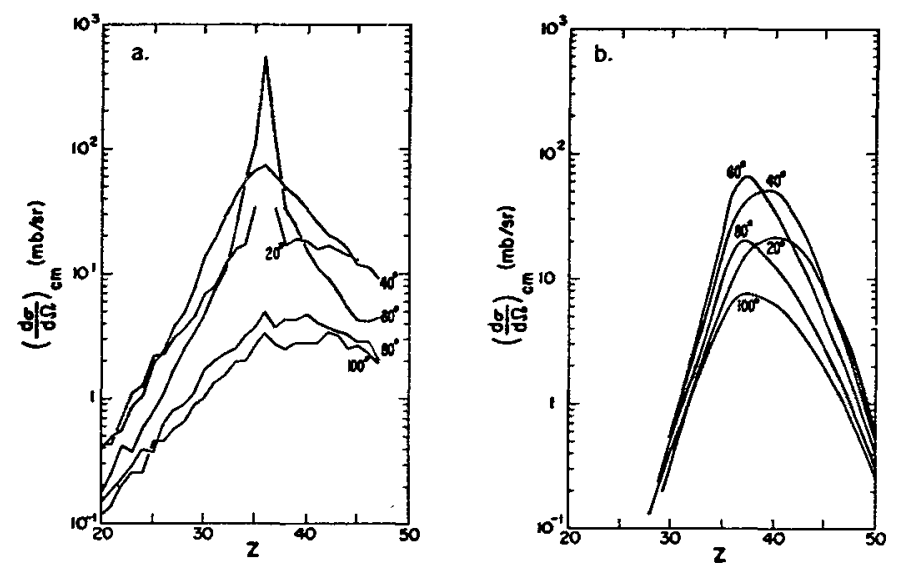

Fig. 35. Comparison between experimental and calculated $Z$ distributions at various center of mass angles for the reaction $197 \mathrm{Au}+86 \mathrm{Kr}$ at $620 \mathrm{MeV}$ bombarding energy [26].

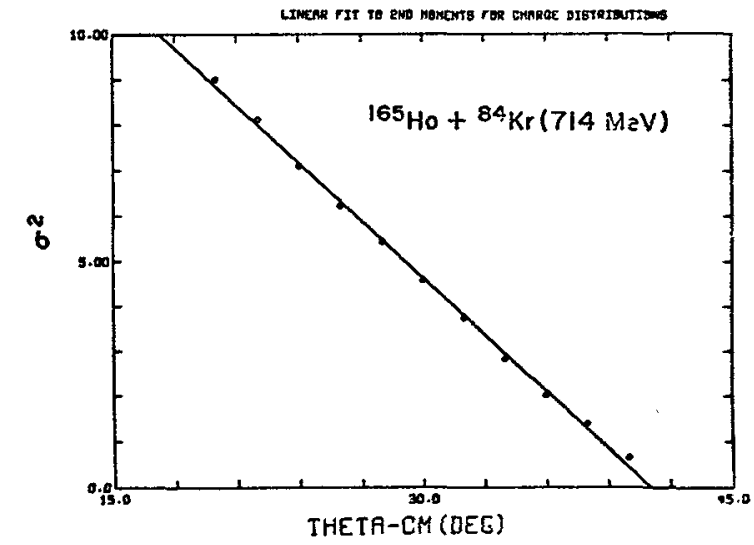

Fig. 36. Variance of the charge distribution $\sigma_{Z}^{2}$ as a function of center of mass angle for the reaction $165 \mathrm{Ho}+{ }^{84} \mathrm{Kr}$ at $714 \mathrm{MeV}$ bombarding energy $[47,59]$.

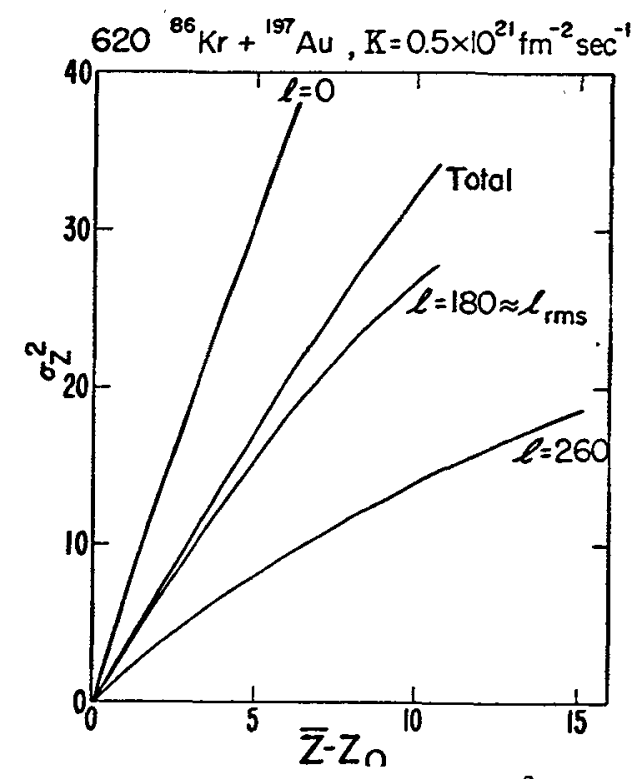

Fig. 37. Calculated dependence of $\sigma_{Z}^{2}$ vs. $Z-z_{0}$ $\left(Z_{0}=36\right)$ for various $\ell$ waves in the reaction $199 \mathrm{Au}+86 \mathrm{Kr}$ at $620 \mathrm{MeV}$ bombarding energy. The $\sigma_{Z}^{2}$ vs. $z-z_{0}$ curve for the distribution ohtained by integrating over $l$ with the $2 l+1$ weight is also shown.

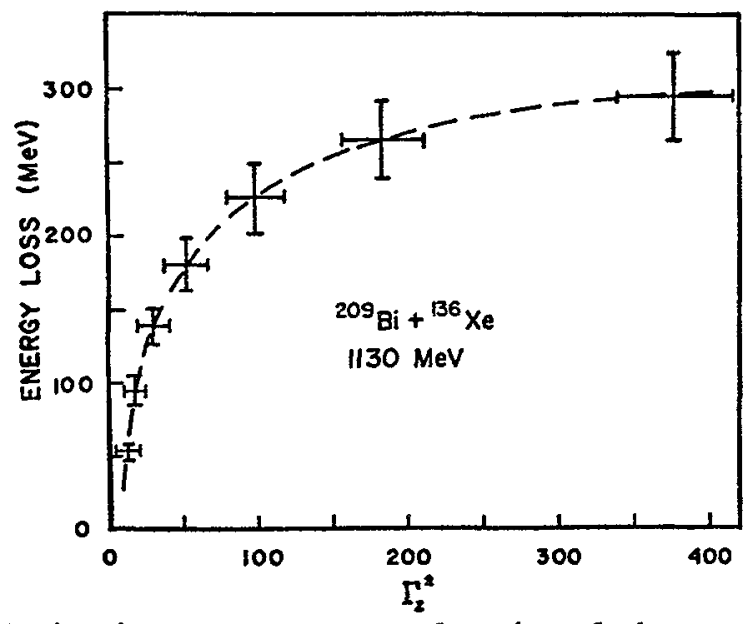

Fig. 38. Experimental kinetic energy loss as a function of the square of the measured $Z$ distribution width (FWHM) for the reaction ${ }^{209} \mathrm{Bi}+{ }^{136} \mathrm{Xe}$ at $1130 \mathrm{MeV}$ bombarding energy $[55,60]$. 